\title{
Monetary Equilibria in a Cash-in-Advance Economy with Incomplete Financial Markets*
}

\author{
Jinhui H. Bai ${ }^{\dagger}$ and Ingolf Schwarz ${ }^{\S}$
}

May 19, 2006

\begin{abstract}
The general equilibrium model with incomplete financial markets (GEI) is extended by adding fiat money, fiscal and monetary policy and a cash-in-advance constraint. The central bank either pegs the interest rate or money supply while the fiscal authority sets a Ricardian or a non-Ricardian fiscal plan. We prove the existence of equilibria in all four scenarios. In Ricardian economies, the conditions required for existence are not more restrictive than in standard GEI. In nonRicardian economies, the sufficient conditions for existence are more demanding. In the Ricardian economy, neither the price level nor the equivalent martingale measure is determinate.

JEL classification: D52; E40; E50

Keywords: Money; Incomplete Markets; Fiscal Policy; Indeterminacy

\footnotetext{
${ }^{*}$ We are grateful to John Geanakoplos, Martin Hellwig and Herakles Polemarchakis for helpful discussions. We benefited from comments of Ruediger Bachmann, Truman Bewley, Gaetano Bloise, Tomoyuki Nakajima, Marek Weretka and the participants in the seminar at the Max Planck Institute for Research on Collective Goods in Bonn, the workshop for mathematical economics at Yale, the 1st Annual CARESS-Cowles Conference on General Equilibrium Theory and its Applications at Yale, the 14th European Workshop on General Equilibrium Theory in Zurich, the GETA 2005 in Tokyo and the 2005 SAET Conference in Vigo. The authors are grateful for the hospitality of the Max Planck Institute for Research on Collective Goods in Bonn and of the Cowles Foundation at Yale, respectively. The usual disclaimer applies.

${ }^{\dagger}$ Corresponding Author: E-mail: jinhui.bai@aya.yale.edu, Phone: +1-203-500-7858, Fax: +1-202-687-6102.

${ }^{\ddagger}$ Department of Economics, Georgetown University, Washington, DC 20057-1036, USA.

${ }^{\S}$ Max Planck Institute for Research on Collective Goods, Kurt-Schumacher-Str. 10, 53113 Bonn, Germany, and CDSEM, University of Mannheim, Germany. E-mail address: ingolf.schwarz@web.de.
} 


\section{Introduction}

In this paper we extend the standard general equilibrium model with incomplete financial markets by introducing fiat money and adding a public authority. The latter consists of a fiscal and a monetary authority. The fiscal authority sets a fiscal plan consisting of taxes, nominal transfers and a debt policy. The monetary authority, or the central bank, creates fiat money at zero costs and earns seignorage from its monetary policy. The actions of both authorities are linked by a common public budget constraint. The transactions technology is supposed to be a simple cash-in-advance constraint. If the nominal interest rates are positive, non-interest bearing fiat money is dominated as a store of value by an interest-bearing nominal bond. The demand for money comes from its role to facilitate trade by means of the cash-in-advance constraint within the states of the economy.

As argued in the Fiscal Theory of the Price Level (See, e.g., Woodford 1995), the introduction of a fiscal authority might add additional restrictions on the set of equilibria. It is well understood that this possibility depends on whether the fiscal policy is of the Ricardian or the non-Ricardian type. Following Woodford (2001), a fiscal policy is called Ricardian if the government budget is satisfied for every price vector. If the budget is valid only for some prices, it is called non-Ricardian. In the latter case, the fiscal policy adds additional restrictions on the equilibrium set. ${ }^{1}$

We study four important combinations of fiscal and monetary policies by combining nominal interest rate peg and money supply policy of the central bank with a Ricardian and a non-Ricardian fiscal policy. For all these cases, we prove existence of an equilibrium and characterize its determinacy properties.

If the fiscal authority follows a Ricardian policy, there exist monetary competitive equilibria under assumptions which are close to the standard assumptions in GEI with financial assets. As in the standard GEI model without a central bank and a fiscal authority, the equilibrium in this Ricardian framework is not determinate. More precisely, there exists a monetary equilibrium under a Ricardian fiscal rule for every fixed positive price level and for every fixed equivalent martingale measure. This result is true for both interest rate peg and money supply policy. Under interest rate peg, we argue that the indeterminacy of the price level is purely nominal but the indeterminacy of the martingale measure can be expected to be real when

\footnotetext{
${ }^{1}$ In the general equilibrium literature, the idea that a non-Ricardian policy might lead to a determinate equilibrium first appeared in Dubey and Geanakoplos (1992). They formally prove the generic local uniqueness under a particular non-Ricardian fiscal policy.
} 
markets are incomplete. Under money supply control, we conjecture that the indeterminacy of the price level might also be real.

If the fiscal authority follows a non-Ricardian policy, existence of equilibrium requires more restrictive assumptions as compared to the Ricardian case. Loosely speaking, the existence of equilibrium requires either high enough gains to trade or positive tax returns. The intuition is that if the fiscal authority fixes nominal transfers at some predetermined and positive level, it must earn seignorage or tax returns to be able to balance its budget. If taxes are zero, then the gains to trade in the economy must be large enough to induce some positive seigniorage income for the government. To make this argument precise, we use the measure for the gains to trade introduced by Dubey and Geanakoplos (1992, 2003a).

Importantly, every obvious degree of indeterminacy we found in the $\mathrm{Ri}$ cardian economy is lost if we assume that the government trades riskless bonds only. Dubey and Geanakoplos (2006) provide a formal proof for generic local uniqueness of equilibria under such a fiscal policy. This result illustrates the role of fiscal policy for the determinacy of the equilibrium.

The main contributions of this paper to the recent literature are the following. First, we show existence and characterize indeterminacy in a cash-in-advance economy with incomplete financial market systems and a Ricardian fiscal policy. ${ }^{2}$ Our results extend the previous findings on existence and indeterminacy in Dréze and Polemarchakis (2000), Bloise et al. (2005), Bloise (2006) and Nakajima and Polemarchakis (2005) under complete markets to incomplete markets. Under a particular non-Ricardian policy, Dubey and Geanakoplos (2003(b)) prove existence of general competitive equilibria under both interest rate peg and money supply control with incomplete markets. They use a strategic market game approach to derive their results. The second contribution of our paper is to provide an alternative proof of existence in a non-Ricardian model. Our method to prove existence adopts more traditional techniques of general equilibrium analysis, and does not rely on a market game analysis.

The paper is organized as follows. In Section 2, we describe the monetary economy including the government and define the general equilibrium. In Section 3, we present our main results for a Ricardian economy, including both interest rate peg and money supply policy. In Section 4, we provide a parallel result for a non-Ricardian economy. In Section 5 we conclude the

\footnotetext{
${ }^{2}$ After completing the first draft of this paper, we learned that Gourdel and Triki (2005) independently studied a similar economy under interest rate peg. They obtain results similar to our Theorems 1 and 3. We will comment on this in Sections 3.3 and 4.3.
} 
paper and give the proofs of all results in the Appendix.

\section{The model}

\subsection{The economy}

We study an exchange economy which extends over two dates, the present time $t=0$ and the future $t=1$. The present is known with certainty, but at date 1 there are $S$ possible states of nature which we index with $s \in S=\{1, \ldots, S\}$. Including the present, there are $S+1$ states of nature lying in the set $S^{*}:=\{0,1, \ldots, S\}$. At every $s \in S^{*}$ there are $L$ consumption goods which are indexed with $l=1, \ldots, L$ and traded at spot prices $p_{s l}$. We denote a consumption plan at state $s \in S^{*}$ with $x_{s}=\left(x_{s 1}, \ldots, x_{s L}\right) \in \mathbb{R}_{+}^{L}$, an overall consumption plan with $x=\left(x_{0}, \ldots, x_{s}, \ldots, x_{S}\right) \in \mathbb{R}_{+}^{(S+1) L}$, a price vector at state $s \in S^{*}$ with $p_{s}=\left(p_{s 1}, \ldots, p_{s L}\right) \in \mathbb{R}_{+}^{L}$ and an overall price vector with $p=\left(p_{0}, \ldots, p_{s}, \ldots, p_{S}\right) \in \mathbb{R}_{+}^{(S+1) L}$. All commodities are perishable.

In $t=0$, there are asset markets for $J \leq S$ financial contracts indexed with $j=1, \ldots, J$. Each asset is a promise to deliver $V_{s}^{j} \in \mathbb{R}_{+}$units of money in every state $s \in S$ and is traded at price $q_{j}$ in period zero. The first asset is assumed to be a nominal riskless government bond. There is no default and each risky financial asset is in zero net supply. Denote the $S \times J$-matrix of returns with $V$, the $S \times(J-1)$-matrix of the returns of the risky assets with $A$, the $1 \times J$-vector of asset prices with $q_{V}$ and the $1 \times(J-1)$-vector of asset prices excluding the price of the bond with $q$. The price of the one period nominal bond between $t=0$ and $t=1$ is $\frac{1}{1+r_{0}}$, where $r_{0}$ is the nominal interest rate between $t=0$ and $t=1$.

We will assume that, within every node $s \in S^{*}$, the asset markets open before the commodity markets and on the commodity markets, a household receives the revenues from selling endowments at the end of the respective node. Therefore, in every state $s \in S$ in $t=1$, a nominal riskless bond can again be traded to allow agents to borrow against their income which they receive at the end of this period. However, there is no uncertainty involved at this stage, i.e., each state $s \in S$ has only one successor state and this state serves for accounting purposes only. The date of these successor states is called accounting period. The price of a bond traded in state $s \in S$ is $\frac{1}{1+r_{s}}$, where $r_{s}$ is the nominal interest rate between state $s \in S$ and the accounting period.

In addition, there is fiat money which can also be held as a store of value 
between $t=0$ and $t=1$ and between $t=1$ and the accounting period. We impose the following general assumption on the structure of the financial assets:

Assumption 1 rank $(V)=J \leq S$. There exists a riskless asset at each $s \in S^{*}$.

\subsection{The households}

The economy is populated by a finite set $I:=\{1, \ldots, I\}$ of households. At $t=0$, the asset markets open first. On these markets, the household trades money $n_{0}^{i} \in \mathbb{R}_{+}$, riskless government bonds $b_{0}^{i} \in \mathbb{R}$ and a portfolio of risky assets $\theta^{i} \in \mathbb{R}^{J-1}$. In addition, household $i$ receives a (lump-sum) transfer $\delta^{i} H_{0}$ from the government, where $H_{0} \in \mathbb{R}_{+}$is the aggregate transfer from which every household $i$ gets a share $\delta^{i} \in \mathbb{R}_{++}$. Therefore, household $i$ faces the constraint

$$
\frac{b_{0}^{i}}{1+r_{0}}+q \cdot \theta^{i}+n_{0}^{i}=\delta^{i} H_{0}
$$

where $\frac{1}{1+r_{0}}$ is the price of the nominal bond. In the goods markets, which open next, household $i$ is subject to the following cash-in-advance constraint: ${ }^{3}$

$$
p_{0} \cdot\left(x_{0}^{i}-e_{0}^{i}\right)^{+} \leq n_{0}^{i} .
$$

The money at the end of $t=0, m_{0}^{i}$, is

$$
\begin{aligned}
m_{0}^{i} & =\left(n_{0}^{i}-p_{0} \cdot\left(x_{0}^{i}-e_{0}^{i}\right)^{+}\right)+p_{0} \cdot\left(x_{0}^{i}-e_{0}^{i}\right)^{-} \\
& =n_{0}^{i}-p_{0} \cdot x_{0}^{i}+p_{0} \cdot e_{0}^{i} .
\end{aligned}
$$

Combining (1) and (3), we get

$$
p_{0} \cdot x_{0}^{i}+\frac{b_{0}^{i}}{1+r_{0}}+q \cdot \theta^{i}+m_{0}^{i}=\delta^{i} H_{0}+p_{0} \cdot e_{0}^{i} .
$$

Equation (4) is the familiar flow budget constraint, which says that the total expenditure within one period cannot exceed the total wealth.

From (2) and (3), we get an equivalent formulation of the cash-in-advance constraint as

$$
m_{0}^{i} \geq p_{0} \cdot\left(x_{0}^{i}-e_{0}^{i}\right)^{-} .
$$

\footnotetext{
${ }^{3}$ We use the usual definition of the negative and the positive part of a vector: $x^{+}:=$ $\left(\ldots, \max \left\{x_{i}, 0\right\}, \ldots\right)$ and $x^{-}:=\left(\ldots, \max \left\{-x_{i}, 0\right\}, \ldots\right)$ so that $x=x^{+}-x^{-}$.
} 
We will use this formulation for the transactions technology because it turns out to be more convenient.

Household $i \in I$ pays a tax $\tau_{s}^{i}$ in state $s \in S^{*}{ }^{4}$ The tax is specified as the market value of a vector of commodities, $\tau_{s}^{i} \in \mathbb{R}_{+}^{L}$, i.e., its budgetary impact is $p_{s} \cdot \tau_{s}^{i}$. The payment of these taxes occurs at the end of state $s \in S^{*}$ in question.

Denoting household $i^{\prime} s$ quantity of a bond traded at the beginning of state $s \in S$ in $t=1$ with $b_{s}^{i}$ and the transfer to household $i$ in state $s \in S$ with $\delta^{i} H_{s}$. The flow budget constraint then reads

$$
p_{s} \cdot x_{s}^{i}+\frac{b_{s}^{i}}{1+r_{s}}+m_{s}^{i}=b_{0}^{i}+A_{s} \cdot \theta^{i}+m_{0}^{i}+\delta^{i} H_{s}+p_{s} \cdot e_{s}^{i}-p_{0} \cdot \tau_{0}^{i}, \forall s \in S .
$$

The cash-in-advance constraint is

$$
m_{s}^{i} \geq p_{s} \cdot\left(x_{s}^{i}-e_{s}^{i}\right)^{-}, \forall s \in S .
$$

In the accounting period following $t=1$, the only economic activity is the payment of the debt and of the income tax in state $s \in S, p_{s} \cdot \tau_{s}^{i}$. Therefore, the terminal condition is

$$
0 \leq b_{s}^{i}+m_{s}^{i}-p_{s} \cdot \tau_{s}^{i}, \forall s \in S .
$$

In the optimal choice, this condition will hold as an equality.

For each $i \in I$, define $e^{i}:=\left(e_{s}^{i}\right)_{s \in S^{*}}, \tau^{i}:=\left(\tau_{s}^{i}\right)_{s \in S^{*}}, m^{i}:=\left(m_{s}^{i}\right)_{s \in S^{*}}$ and $b^{i}:=\left(b_{s}^{i}\right)_{s \in S^{*}}$. The budget set of every household $i$ is the set ${ }^{5}$

$B^{i}(p, q, r, H):=\left\{\left(x^{i}, m^{i}, b^{i}, \theta^{i}\right) \in \mathbb{R}_{+}^{(S+1) L} \times \mathbb{R}_{+}^{S+1} \times \mathbb{R}^{S+1} \times \mathbb{R}^{J-1} \mid(4)-(8)\right.$ hold $\}$.

Every household $i \in I$ gets utility from consuming in every node $s \in$ $S^{*}$ according to a function $u^{i}: \mathbb{R}_{+}^{(S+1) L} \rightarrow \mathbb{R}$. We make the following assumptions on the household sector:

Assumption 2 For each consumer $i$, the utility function $u^{i}$ is continuous, quasi-concave and strictly increasing.

\footnotetext{
${ }^{4}$ We emphasize that taxes are only included to make the model more general. Not a single argument, neither related to the existence of a monetary equilibrium nor related to the (in)determinacy, depends on strictly positive taxes.

${ }^{5}$ To save the notation, we suppress the parameters in the notation. The budget set should always be understood as $B^{i}(p, q, r, H):=B^{i}\left(p, q, r, H ; e^{i}, \tau^{i}, \delta^{i}\right)$.
} 
Assumption 3 Every household has some endowments after tax in every state, i.e., $\forall i \in I,\left(e_{s}^{i}-\tau_{s}^{i}\right)>0$ for every $s \in S^{*}$. Household one has strictly positive endowments after tax at every node, i.e., $\left(e^{1}-\tau^{1}\right) \gg 0$. Aggregate endowments are bounded, i.e., $\sum_{i}\left(e^{i}-\tau^{i}\right) \ll+\infty{ }^{6}$

\subsection{The government}

At each state $s \in S^{*}$, the government taxes the household and distributes transfers. We denote the total commodity tax by $\tau:=\left(\tau_{s}\right)_{s \in S^{*}} \in \mathbb{R}_{+}^{(S+1) L}$, where $\tau_{s}:=\sum_{i=1}^{I} \tau_{s}^{i}$. The total lump-sum transfer is the vector $H:=$ $\left(H_{s}\right)_{s \in S^{*}} \in \mathbb{R}_{+}^{S+1}$. For simplicity, we assume throughout the paper that the transfer is distributed according to the shares $\left(\delta^{i}\right)_{i \in I}, \sum_{i} \delta^{i}=1$.

The government trades riskless bonds $B=\left(B_{s}\right)_{s \in S^{*}}$ and supplies balances $M=\left(M_{s}\right)_{s \in S^{*}}$. If $B_{s}>0$ then the government sells bonds and hence the term represents new indebtedness against the private sector. If $B_{s}<0$, it means the loan to the private sector.

Assumption 4 The government only trades riskless bonds.

This assumption can be justified by an appeal to realism. It has consequences for the determinacy of equilibria. We will comment on this in Section 4.3 .

It follows that the government budget constraint is

$$
\frac{B_{0}}{1+r_{0}}+M_{0}=H_{0}
$$

in period zero and

$$
\frac{B_{s}}{1+r_{s}}+M_{s}+p_{0} \cdot \tau_{0}=B_{0}+M_{0}+H_{s}, \forall s \in S
$$

in period one. Both (9) and (10) need to hold for every vector of commodity price and interest rates.

In the accounting period following each state $s \in S$, in equilibrium it must be true that

$$
B_{s}+M_{s}-p_{s} \cdot \tau_{s}=0, \forall s \in S
$$

\footnotetext{
${ }^{6} \mathrm{~A}$ vector $x \in \mathbb{R}^{n}$ satisfies $x>0$ if and only if $x_{i} \geq 0, \forall i=1, \ldots, n$, and if there is a $j$ such that $x_{j}>0$. Accordingly, $x \gg 0$ if and only if $x_{i}>0, \forall i=1, \ldots, n$ and $x \geq 0$ if and only if $x_{i} \geq 0, \forall i=1, \ldots, n$.
} 
In fact, because preferences are monotone, households choose their plans so that there is no slack in (8). In the optimal choice of households, one therefore has $\sum_{i \in I}\left(b_{s}^{i}+m_{s}^{i}-p_{s} \cdot \tau_{s}^{i}\right)=0$ for all $s \in S$. Since market clearing requires $\sum_{i \in I} b_{s}^{i}=B_{s}$ and $\sum_{i \in I} m_{s}^{i}=M_{s}$, one gets (11). We should emphasize that this is only one way to derive (11). An alternative approach would postulate (11) directly as the government's budget constraint for the accounting period. This would be appropriate if money was a kind of debt of the government so that, in fact, the government is under the legal obligation to withdraw the money that is issued from the system. For "outside" money, which involves no obligation, this reasoning does not apply.

Whether (11) holds for off-equilibrium situations depends on the nature of the fiscal policy. Indeed, a Ricardian fiscal authority adjusts its actions such that (11) holds for whatever vector of prices and money supply prevailing in the economy. Hence it is an identity rather than an equilibrium restriction. A non-Ricardian fiscal authority determines (at least a part of) its policy instruments such that (11) is not true for some price vectors and money supply. To make these points precise, we denote a fiscal policy rule as $(H, B)(p, M, r)$, i.e., a plan for transfers and bond market actions contingent on the commodity prices, money supply and interest rates, ${ }^{7}$ and give the following definition.

Definition 1 A fiscal policy rule $(H, B)(p, M, r)$ is called Ricardian if equation (9)-(11) holds for every $(p, M, r) \in \mathbb{R}_{+}^{(S+1) L} \times \mathbb{R}_{+}^{S+1} \times \mathbb{R}_{+}^{S+1}$. If there exists $\left(p^{*}, M^{*}, r^{*}\right) \in \mathbb{R}_{+}^{(S+1) L} \times \mathbb{R}_{+}^{S+1} \times \mathbb{R}_{+}^{S+1}$ such that $(H, B)\left(p^{*}, M^{*}, r^{*}\right)$ does not satisfy (9)-(11), then it is called non-Ricardian.

As we shall see later, whether a fiscal policy is of Ricardian or nonRicardian type has important implication for the determinacy of equilibrium. Consequently, we will study both a Ricardian fiscal policy characterized by endogenous transfers and a non-Ricardian fiscal policy characterized by exogenous transfers. This leads to four different combinations of fiscal and monetary policy of the government: the central bank might peg the interest rate or fix the money supply, while the fiscal authority might determine transfers endogenously or fix them exogenously.

In the case of endogenous transfers, the fiscal authority redistributes the seigniorage income and the tax returns at each state of the economy. The

\footnotetext{
${ }^{7}$ In general, the fiscal policy consists of a plan for taxes, transfers and bond market actions. However, in this paper we keep the taxes fixed and restrict attention to different transfer policies in combination with bond market actions.
} 
government bonds adjust accordingly to satisfy the constraints of the government. Bloise and Polemarchakis (2006) call such a policy a balanced transfer rule. We adopt their terminology and say that the fiscal policy follows a balanced transfer rule if it satisfies the following definition:

Definition 2 The balanced transfer fiscal policy determines the vector $(H, B)$ by the functions $H(p, M, r)$ and $B(p, M, r)$, where

$$
\begin{aligned}
H_{s}(p, M, r) & :=\frac{r_{s}}{1+r_{s}} M_{s}+\frac{p_{s} \cdot \tau_{s}}{1+r_{s}}, \forall s \in S^{*}, \\
B_{s}(p, M, r) & :=p_{s} \tau_{s}-M_{s}, \forall s \in S^{*} .
\end{aligned}
$$

Under the balanced transfer policy, one can check that equation (9)(11) always holds. Therefore, this fiscal policy is Ricardian. The balanced transfer rule says that the government distributes its revenue in every state of the world. Hence the government needs to know the value of its seigniorage and the market value of its tax returns at the time when the transfers are distributed, i.e., in the first subperiod within a state since the transfers occur in the asset markets. ${ }^{8}$

A fiscal policy which fixes transfers in every state of the world exogenously will be called fixed transfer fiscal policy. Formally,

Definition 3 The fixed transfer fiscal policy determines the vector $(H, B)$ by the functions $H(p, M, r)$ and $B(p, M, r)$, where

$$
\begin{aligned}
& H_{s}(p, M, r):=\bar{H}_{s}, \forall s \in S^{*}, \text { where } \bar{H}_{0}>0, \bar{H}_{s} \geq 0, \forall s \in S, \\
& B_{0}(p, M, r):=\left(1+r_{0}\right)\left(\bar{H}_{0}-M_{0}\right) \\
& B_{s}(p, M, r):=\left(1+r_{s}\right)\left(B_{0}+M_{0}+\bar{H}_{s}-M_{s}-p_{0} \cdot \tau_{0}\right), \forall s \in S .
\end{aligned}
$$

Importantly, notice that under this policy the equation (11) does not hold for some price and interest rate vector. Therefore, the fixed transfer fiscal policy is non-Ricardian.

\footnotetext{
${ }^{8}$ Since commodity prices are determined on commodity markets which, however, meet when the asset markets are already closed, the specification of such a policy involves an informational problem. Perhaps the most consistent interpretation is, first, that the asset and the commodity markets in fact meet at the same time but at different places and, second, that the possible money flow is restricted to the direction from the asset to the commodity markets. The first point implies that the government can observe the commodity prices for determining its policy, hence resolving the informational problem. The second point implies that the households can use their asset market funds in the commodity markets, as required.
} 


\subsection{Competitive equilibria}

The market clearing condition is specified in the usual way as

$$
\begin{aligned}
\sum_{i=1}^{I} e_{s}^{i} & =\sum_{i=1}^{I} x_{s}^{i}, \quad \forall s \in S^{*}, \\
M_{s} & =\sum_{i=1}^{I} m_{s}^{i}, \quad \forall s \in S^{*}, \\
B_{s} & =\sum_{i=1}^{I} b_{s}^{i}, \quad \forall s \in S^{*}, \\
0 & =\sum_{i=1}^{I} \theta_{s}^{i}, \quad \forall s \in S,
\end{aligned}
$$

where the equation (12), (13), (14), and (15) are commodity, money and asset market clearing conditions, respectively. We write the bond and the risky assets separately since we want to emphasize the difference of the market supply in these two cases.

The primitives of the economy can be summarized by the vector

$$
\mathcal{E}:=\left\{\left(u^{i}, e^{i}, \tau^{i}, \delta^{i}\right)_{i \in I}, V\right\} .
$$

Definition 4 An equilibrium for the economy $\mathcal{E}$ is a tuple

$$
\left\{(\bar{p}, \bar{q}, \bar{r}),\left(\bar{x}^{i}, \bar{m}^{i}, \bar{b}^{i}, \bar{\theta}^{i}\right)_{i \in I},(\bar{M}, \bar{B}, \bar{H})\right\}
$$

such that

(1) $\left(\bar{x}^{i}, \bar{m}^{i}, \bar{b}^{i}, \bar{\theta}^{i}\right)$ maximizes $u^{i}\left(x^{i}\right)$ subject to $\left(x^{i}, m^{i}, b^{i}, \theta^{i}\right) \in B^{i}(\bar{p}, \bar{q}, \bar{r}, \bar{H})$.

(2) The actions of the monetary-fiscal authority $(\bar{M}, \bar{B}, \bar{H})$ satisfy (9), (10) and (11).

(3) In every state, markets clear, i.e., (12)-(15) hold.

An equilibrium is said to be monetary if $\bar{p}_{s l}<+\infty, \forall s \in S^{*}, l \in L$.

In the proofs of the theorems given in the following sections, we use another equilibrium concept which is more tractable for our purposes. It is well known that agent $i$ 's maximization problem has a solution only if there is no arbitrage possibility on the financial markets. Using the results from 
Harrison and Kreps (1979), this implies the existence of a strictly positive probability measure $\mu=\left\{\mu_{s}\right\}_{s \in S}$ such that $q_{V}=\frac{\mu}{1+r_{0}} V . \mu$ is called the equivalent martingale measure.

Denote an $S$-dimensional vector $\left(v_{1}, \ldots, v_{S}\right)$ by $v_{\mathbf{1}}$. In addition, $\frac{r_{1}}{1+r_{1}}:=$ $\left(\frac{r_{s}}{1+r_{s}}\right)_{s \in S}$ and $\frac{1}{1+r_{1}}:=\left(\frac{1}{1+r_{s}}\right)_{s \in S}$. Combine (8) and (6) to get, $\forall s \in S$,

$$
p_{s} \cdot x_{s}^{i}+\frac{p_{s} \cdot \tau_{s}^{i}}{1+r_{s}}+\frac{r_{s}}{1+r_{s}} m_{s}^{i}=b_{0}^{i}+A_{s} \cdot \theta^{i}+m_{0}^{i}+\delta^{i} H_{s}+p_{s} \cdot e_{s}^{i}-p_{0} \cdot \tau_{0}^{i}
$$

Substitute the no-arbitrage condition $q_{V}=\left(\frac{1}{1+r_{0}}, q_{2}, \ldots, q_{J}\right)=\frac{\mu}{1+r_{0}} V=$ $\frac{\mu}{1+r_{0}}\left(\mathbf{1}, A_{1}, \ldots, A_{J-1}\right)^{\prime 9}$ into (4) and plug in (16) for each $s \in S$ to get

$$
\begin{aligned}
p_{0} & \cdot x_{0}^{i}+\frac{r_{0}}{1+r_{0}} m_{0}^{i}+\frac{\mu}{1+r_{0}} \cdot\left(p_{\mathbf{1}}^{\square} \square x_{\mathbf{1}}^{i}+\frac{r_{\mathbf{1}}}{1+r_{\mathbf{1}}} \square m_{\mathbf{1}}^{i}\right)=\delta^{i} H_{0} \\
& +p_{0} \cdot\left(e_{0}^{i}-\frac{\tau_{0}^{i}}{1+r_{0}}\right)+\frac{\mu}{1+r_{0}} \cdot\left(\delta^{i} H_{\mathbf{1}}+p_{\mathbf{1}} \square\left(e_{\mathbf{1}}^{i}-\frac{1}{1+r_{\mathbf{1}}} \square \tau_{\mathbf{1}}^{i}\right)\right),
\end{aligned}
$$

where $m \square n:=\left(m_{s} \cdot n_{s}\right)_{s \in S}$. The left hand side is the expenditure in terms of its date-0 value, while the right hand side is the discounted nominal wealth. For household 1, define the complete markets budget set

$$
\bar{B}^{1}(p, \mu, r, H)=\left\{\left(x^{1}, m^{1}\right) \in \mathbb{R}_{+}^{(S+1) L} \times \mathbb{R}_{+}^{S+1} \mid(5),(7),(17) \text { hold. }\right\} .
$$

From the no-arbitrage conditions, the budget sets of agents $i \geq 2$ can also be expressed as depending on $\mu$ instead of $q$. Following Cass (1984) and Duffie and Shafer (1985), in Definition 5 we define a concept of effective monetary equilibrium.

Definition 5 An effective equilibrium for the economy $\mathcal{E}$ is a tuple

$$
\left\{(\bar{p}, \bar{\mu}, \bar{r}),\left(\bar{x}^{i}, \bar{m}^{i}, \bar{b}^{i}, \bar{\theta}^{i}\right)_{i \in I},(\bar{M}, \bar{B}, \bar{H})\right\}
$$

such that

(1) For $i \geq 2,\left(\bar{x}^{i}, \bar{m}^{i}, \bar{b}^{i}, \bar{\theta}^{i}\right)$ maximizes $u^{i}\left(x^{i}\right)$ subject to $\left(x^{i}, m^{i}, b^{i}, \theta^{i}\right) \in$ $B^{i}(\bar{p}, \bar{\mu}, \bar{r}, \bar{H})$. For $i=1,\left(\bar{x}^{1}, \bar{m}^{1}\right)$ maximizes $u^{1}\left(x^{1}\right)$ such that $\left(x^{1}, m^{1}\right) \in$ $\bar{B}^{1}(\bar{p}, \bar{\mu}, \bar{r}, \bar{H})$, and $\left(\bar{b}^{1}, \bar{\theta}^{1}\right)=\left(\bar{B}-\sum_{i=2}^{I} \bar{b}^{i},-\sum_{i=2}^{I} \bar{\theta}^{i}\right)$.

\footnotetext{
${ }^{9}$ We use the notation $1:=(\ldots, 1, \ldots)$.
} 
(2) The actions of the monetary-fiscal authority $(\bar{M}, \bar{B}, \bar{H})$ satisfy (9), (10) and (11).

(3) In every state, commodity and money markets clear, i.e., (12)-(13) hold.

An effective equilibrium is said to be monetary if $\bar{p}_{s l}<+\infty, \forall s \in S^{*}, l \in$ $L$.

From Definitions 4 and 5, we can immediately see two differences. First, in the effective equilibrium, household 1 is only restricted by the intertemporal budget constraint and the cash-in-advance constraint. Second, household 1 does not choose $\left(\bar{b}^{1}, \bar{\theta}^{1}\right)$ directly. Instead he chooses a bond demand to clear the bond markets and a demand for the risky assets to clear these asset markets.

It is immediate that every effective equilibrium corresponds to an equilibrium as defined in Definition 4. Indeed, it is easy to see that the noarbitrage conditions determine $\bar{q}$ given $\bar{\mu}$ and $\bar{r}$. To show that a tuple $\left\{(\bar{p}, \bar{\mu}, \bar{r}),\left(\bar{x}^{i}, \bar{m}^{i}, \bar{b}^{i}, \bar{\theta}^{i}\right)_{i \in I},(\bar{M}, \bar{B}, \bar{H})\right\}$ as defined in the effective monetary equilibrium corresponds to a monetary equilibrium, we first need to check that the household 1 satisfies the budget equations (4) - (8) and second that his choice is still optimal in the sequential constraint. The first property follows directly from Walras law. ${ }^{10}$ To see that household one still maximizes his utility, just notice that the sequential constraint is a subset of the intertemporal one. Hence, the old consumption vector must be optimal since it is still feasible under the sequential constraint and it was already optimal in the larger intertemporal constraint. These arguments are standard and not made explicit here.

\section{Monetary equilibria with balanced transfers}

\subsection{Interest rate peg}

If the central bank pegs the nominal interest rate, then the vector $r:=$ $\left\{r_{s}\right\}_{s \in S^{*}}$ is fixed at a target value $\bar{r}$. To sustain $\bar{r}$ in the market, the central bank accommodates money demand. We impose the following assumption on monetary policy:

Assumption 5 Interest rates are nonnegative and bounded above, $0 \leq \bar{r}_{s}<$ $+\infty, \forall s \in S^{*}$, and the government accommodates money demand, i.e., $M_{s}=$ $\sum_{i} m_{s}^{i}$ for each $s \in S^{*}$.

\footnotetext{
${ }^{10}$ We leave it an exercise to the reader to check these equations.
} 
A monetary equilibrium with interest rate peg and balanced transfers can now be defined as follows:

Definition 6 A monetary equilibrium with interest rate peg and balanced transfers is a monetary equilibrium according to Definition 4 with exogenously fixed $r$ satisfying Assumption 5 and a fiscal policy rule according to Definition 2.

In the following theorem, we show that for every fixed price level and for every fixed martingale measure, there exists a monetary equilibrium which implements the interest rate target of the central bank.

Theorem 1 Suppose Assumptions 1 - 5 hold. Fix $0<\bar{c}<+\infty$ and $\bar{\mu} \gg 0$, then for every $0 \leq \bar{r} \ll+\infty$ there exists a monetary equilibrium with interest rate peg and balanced transfers $\left\{(\bar{p}, \bar{q}, \bar{r}),\left(\bar{x}^{i}, \bar{m}^{i}, \bar{b}^{i}, \bar{\theta}^{i}\right)_{i \in I},(\bar{M}, \bar{B}, \bar{H})\right\}$ such that $\bar{c}=\sum_{l} \bar{p}_{0 l}+\sum_{l \in L, s \in S} \bar{\mu}_{s} \bar{p}_{s l}$ and $\bar{q}=\frac{\bar{\mu}}{1+\bar{r}_{0}} A$.

\subsection{Money supply control}

Under money supply control, the central bank fixes the money supply process $M:=\left(M_{s}\right)_{s \in S^{*}}$ at a target value $\bar{M}$. If this is the case, we impose

Assumption 6 Under money supply policy, $0<\bar{M}_{s}<+\infty, \forall s \in S^{*}$.

Combining the balanced transfer policy with money supply control suggests the following definition:

Definition 7 A monetary equilibrium with money supply control and balanced transfers is a monetary equilibrium according to Definition 4 with exogenously fixed $M$ satisfying Assumption 6 and a fiscal policy rule according to Definition 2.

In the next theorem we show that for every fixed price level and for every fixed martingale measure, there exists a monetary equilibrium which implements a money supply target $\bar{M}$ of the central bank. So the result parallels the result from the previous theorem under interest rate peg policy.

Theorem 2 Suppose Assumptions $1-4$ and 6 hold. Fix $0<\bar{c}<+\infty$ and $\bar{\mu} \gg 0$, then for every $0 \ll \bar{M} \ll+\infty$ there exists a monetary equilibrium with money supply control and balanced transfers $\left\{(\bar{p}, \bar{q}, \bar{r}),\left(\bar{x}^{i}, \bar{m}^{i}, \bar{b}^{i}, \bar{\theta}^{i}\right)_{i \in I}\right.$, $(\bar{M}, \bar{B}, \bar{H})\}$ such that $\bar{c}=\sum_{l} \bar{p}_{0 l}+\sum_{l \in L, s \in S} \bar{\mu}_{s} \bar{p}_{s l}$ and $\bar{q}=\frac{\bar{\mu}}{1+\bar{r}_{0}} A$. 


\subsection{Interpretation and literature}

We provide some intuition for the existence and the indeterminacy results in Theorems 1 and 2. To prove existence of an equilibrium we use similar assumptions as in the GEI-model with nominal assets. The balanced transfer rule implies that (11) is true both in and out of equilibrium. Hence this condition is an identity which does not add additional restrictions as compared to the standard GEI. This explains why we do not need additional assumptions even in a more complex model with a government and the balanced transfer fiscal policy. Note that our equilibrium could be a no-trade equilibrium in which there is no seigniorage income for the central bank. In this case, the government just redistributes potential tax returns among the households according to their shares $\left(\delta^{i}\right)_{i \in I}$.

The intuition concerning indeterminacy can be given by counting equations and variables. The macro variables to be determined in the effective equilibrium are the $L(S+1)$ commodity spot prices, the $S-1$ dimensional equivalent martingale measure and the $S+1$ interest rates. There are $L(S+1)$ equilibrium restrictions coming from commodity market clearing and $S+1$ money market clearing equations. Under interest rate peg, the latter $S+1$ equations are identities and the $S+1$ interest rates are fixed exogenously, hence both the equations and the variables cannot be counted. Finally, there is a single Walras law at work. To see this, note that in every effective equilibrium, (9), (10) and (11) hold. From this, it is not hard to see that, in every effective equilibrium,

$$
\begin{aligned}
\frac{r_{0}}{1+r_{0}} M_{0}+\frac{p_{0} \cdot \tau_{0}}{1+r_{0}} & +\frac{\mu}{1+r_{0}} \cdot\left(\frac{r_{\mathbf{1}}}{1+r_{\mathbf{1}}} \square M_{\mathbf{1}}+\frac{1}{1+r_{\mathbf{1}}} \square\left(p_{\mathbf{1}} \square \tau_{\mathbf{1}}\right)\right) \\
& =H_{0}+\frac{\mu}{1+r_{0}} \cdot H_{\mathbf{1}} .
\end{aligned}
$$

From this equation and the fact that household one only faces the intertemporal budget constraint, we easily infer that one Walras Law is at work. In total, there are $S$ more variables than independent equations, so $S$ is the degree of total indeterminacy under both interest rate peg and money supply control. ${ }^{11}$

\footnotetext{
${ }^{11}$ The same intuition can be given for the monetary equilibrium according to Definition 4. Under interest rate peg, the macro variables to be determined are the commodity prices and the prices for the risky assets, which is an $L(S+1)+(J-1)$-dimensional vector. We have $L(S+1)+(J-1)$ market clearing equations for the commodity and the risky asset markets and $(S+1)$ market clearing equations for the bond markets. Including the government budget constraint at every node $s \in S^{*}$, it follows that there are $S+1$
} 
Notice that our results do not rely on the number of assets in the economy. Hence, the same intuition as given above applies for the case of complete markets. ${ }^{12}$ It is also important to point out that neither a Ricardian fiscal policy nor the market clearing for the risky assets plays a role for counting equations and variables in the previous paragraph. This has important implications for the situation when a Ricardian government has access to all the risky assets as well as riskless bonds. As long as the government does not control the price of a risky asset, it seems to be reasonable to conjecture that we will still get the same number of indeterminacy in terms of nominal variables..$^{13}$

Given the $S$-dimensional indeterminacy in terms of nominal variables, it is natural to investigate its implication for the real indeterminacy. In the following two paragraphes, we provide some discussions and conjectures on this issue. We emphasize that our conjectures need to be confirmed formally in a separate paper.

Under interest rate peg, among the $S$ dimensions of indeterminacy there is (at least) one degree of homogeneity involved. Indeed, if agents react to a doubling of the commodity prices by doubling their portfolios and money demand, the transfers and money supply will also double by the balanced transfer rule and money supply adjustment. Hence the allocation is unaffected. This degree of homogeneity on the price level is independent of the number of assets in the economy, as in the standard GEI economy. Whether the remaining $S-1$ degrees of indeterminacy captured by the measure are real depends on the market structure. If financial markets are complete, these $S-1$ degrees of indeterminacy are purely nominal, as argued in Bloise et al. (2005). However, when financial markets are incomplete, it seems reasonable to conjecture that the remaining $S-1$ degrees of indeterminacy captured by the measure are real. An argument which supports this conjecture is given in Nakajima and Polemarchakis (2001). To conclude, we conjecture that the real indeterminacy under interest rate peg and balanced

Walras laws. The balanced transfer rule implies that (11) is always true. From this, we infer another $S$ degrees of redundancies. Hence, in total there are $S$ more variables than equations, which suggests an overall indeterminacy of degree $S$. Under money supply control, there are $S+1$ more variables and equations which clearly leaves the conclusion unaffected.

${ }^{12}$ This is why we get basically the same number of indeterminacy as in the previous literature with complete markets (see Bloise, Dréze and Polemarchakis, 2005; Dréze and Polemarchakis, 2000).

${ }^{13}$ Obviously, if the Ricardian government controls the price of the risky assets directly, it adds additional restrictions to the equivalent martingale measure through no-arbitrage requirement. 
transfer rule takes the same form as in the standard GEI with nominal assets. ${ }^{14}$

Under money supply control, the real indeterminacy could take a different form. First, the degree of homogeneity on the price level is lost in the case of money supply control. Scaling the overall price level cannot be compensated by scaling money demand accordingly since equilibrium money supply is fixed. Hence the indeterminacy captured by the price level might be real. Similarly, a changing price level might change the endogenous transfers which could also imply real effects. Second, a change in the equivalent martingale measure could also lead to real effects under money supply control. With incomplete markets, a similar argument as under interest rate peg could still apply. More importantly, even with complete markets, real effects due to a changing martingale measure are still possible. In fact, because the transfer may not respond to the change in the equivalent martingale measure due to fixed money supply, a different measure could change the discounted value of the transfer (see equation (17)), which may lead to real effects. We conclude this short discussion by conjecturing that the real indeterminacy of Ricardian equilibria with nominal assets, cash-in-advance constraints and money supply control might be different from the standard GEI with nominal assets.

The recent literature on Ricardian economies can be summarized as follows. Dréze and Polemarchakis (2000) and Bloise et al. (2005) prove existence and indeterminacy under interest rate peg with complete asset markets under a finite and an infinite horizon, respectively. Bloise (2006) shows similar results under an infinite horizon with money supply policy. We extend this recent literature on Ricardian economies by proving existence and indeterminacy under both interest rate peg and money supply policy with incomplete markets and a finite time horizon.

Under interest rate peg policy, Gourdel and Triki (2005) independently studied a closely related economy. They obtained a result similar to our Theorem 1. Within the interest rate peg policy, there are two major differences between Gourdel and Triki (2005) and our model. First, in our model the asset markets open before the commodity markets, as in Woodford (1994) and Bloise et al. (2005). In Gourdel and Triki (2005), the bond market opens before the commodity market, but the latter opens before the markets for the risky assets. Second, we use different techniques to prove our results. In our proof we use a trick introduced by Cass (1984), while they

\footnotetext{
${ }^{14}$ The degree of real indeterminacy in the GEI economy with nominal assets was studied by Balasko and Cass (1989) and Geanakoplos and Mas-Colell (1989).
} 
use the method similar to Werner (1985). Our method leads us to characterize the indeterminacy in terms of the total price level and the equivalent martingale measure, while they use the price level within each state as the indeterminate variables.

\section{Monetary equilibria with fixed transfers}

In every equilibrium with fixed transfers, (11) must be true. Plug (11) as a function of $B_{s}$ and (9) as a function of $B_{0}$ into equation (10) to get

$$
\frac{r_{s}}{1+r_{s}} M_{s}+r_{0} M_{0}+\frac{p_{s} \cdot \tau_{s}}{1+r_{s}}+p_{0} \cdot \tau_{0}=\left(1+r_{0}\right) H_{0}+H_{s}, \quad \forall s \in S .
$$

This equation is a necessary condition for an equilibrium under fixed transfers. Notice that it can only be satisfied if either taxes or seigniorage are strictly positive. Under zero taxes, the gains to trade in the economy must hence be large enough to induce some positive seigniorage income for the government.

In the proof of the next two Theorems we will impose a gains to trade hypothesis which goes back to Dubey and Geanakoplos (1992, 2003(a), 2003(b)). Define the function $\bar{\zeta}_{s}: \mathbb{R}^{L} \times \mathbb{R}_{+} \rightarrow \mathbb{R}^{L}$ by

$$
\bar{\zeta}_{s l}\left(\zeta_{s}, \gamma\right):=\left\{\begin{array}{ll}
\zeta_{s l} & \text { if } \zeta_{s l}<0 \\
\frac{\zeta_{s l}}{1+\gamma} & \text { otherwise }
\end{array}, \forall l \in L\right.
$$

A feasible allocation $\left(x_{-s}, e_{s}\right):=\left(x_{0}, x_{1}, \ldots, x_{S}\right)_{\mid x_{s}=e_{s}}$ is said to be $\gamma$-Pareto optimal in state $s \in S$ at $e_{s}$ if there does not exist a trade vector $\zeta_{s} \in \mathbb{R}^{I L}$ in state $s$ such that $\sum_{i} \zeta_{s}^{i}=0$ and, $\forall i \in I, e_{s}^{i}+\zeta_{s}^{i} \geq 0$ and $u^{i}\left(x_{0}^{i}, x_{1}^{i}, \ldots, e_{s}^{i}+\right.$ $\left.\bar{\zeta}_{s}\left(\zeta_{s}^{i}, \gamma\right), \ldots, x_{S}^{i}\right) \geq u^{i}\left(x_{0}^{i}, x_{1}^{i}, \ldots, e_{s}^{i}, \ldots, x_{S}^{i}\right)$ with at least one $i \in I$ where the strict inequality holds. If $\left(x_{-s}, e_{s}\right)$ is $\gamma$-Pareto optimal in state $s \in S$ at $e_{s}$, then we equivalently say that there are no gains to $\gamma$-diminished trade in $s \in S$ at $\left(x_{-s}, e_{s}\right)$. Accordingly, the gains to trade at $\left(x_{-s}, e_{s}\right)$ are defined by

$$
\gamma_{s}\left(x_{-s}, e_{s}\right):=\min \{\gamma \mid \text { there are no gains to } \gamma \text {-diminished trade in } s \in S\} .
$$

\subsection{Interest rate peg}

In the fixed transfer case, we assume that the interest rates are strictly positive. 
Assumption 7 Interest rates are strictly positive and bounded above, $0<$ $\bar{r}_{s}<+\infty, \forall s \in S^{*}$. The government accommodates money demand, i.e., $M_{s}=\sum_{i} m_{s}^{i}$ for each $s \in S^{*}$.

Combining an interest rate peg policy of the central bank with the fixed transfer fiscal policy suggests the following definition:

Definition 8 A monetary equilibrium with interest rate peg and fixed transfers is a monetary equilibrium according to Definition 4 with exogenously fixed interest rates according to Assumption 7 and a fiscal policy rule according to Definition 3.

To rule out an exploding commodity price path, we need to impose either a strictly positive taxation or a gains to trade hypothesis. The following assumption says that if the tax in some state $s \in S$ is zero, then the gains to trade in this state exceed the interest rate. Intuitively, the friction caused by the transactions technology still allows for Pareto-improvements at the initial endowment allocation in the state $s \in S$.

Assumption 8 For every $s \in S$, either $\tau_{s}>0$, or $\gamma_{s}\left(x_{-s}, e_{s}\right)>\bar{r}_{s}$ for all feasible $\left(x_{-s}, e_{s}\right)$.

The following theorem states that every interest rate target of the central bank can be embedded in an equilibrium with fixed transfers. Note that we do not claim any indeterminacy result here.

Theorem 3 Suppose that Assumptions 1 - 4, 7 and 8 hold. For every $0 \ll \bar{r} \ll+\infty$ there exists a monetary equilibrium with interest rate peg and fixed transfers $\left\{(\bar{p}, \bar{q}, \bar{r}),\left(\bar{x}^{i}, \bar{m}^{i}, \bar{b}^{i}, \bar{\theta}^{i}\right)_{i \in I},(\bar{M}, \bar{B}, \bar{H})\right\}$.

\subsection{Money supply control}

The definition of equilibrium is straightforward:

Definition 9 A monetary Equilibrium with money supply control and fixed transfers is a monetary equilibrium according to Definition 4 with exogenously fixed money supply satisfying Assumption 6 and a fiscal policy rule according to Definition 3.

For the same reason as in the interest rate peg, we also need to impose a Gains-to-Trade hypothesis for money supply policy. 
Assumption 9 For every $s \in S$, either $\tau_{s}>0$, or, for every feasible $\left(x_{-s}, e_{s}\right), \gamma_{s}\left(x_{-s}, e_{s}\right)>\frac{\bar{H}_{0}+\bar{H}_{s}}{\bar{M}_{s}-\bar{H}_{0}-\bar{H}_{s}}$ together with $\bar{M}_{0} \geq \bar{H}_{0}$ and $\bar{M}_{s}>$ $\bar{H}_{0}+\bar{H}_{s}$.

The last theorem states the parallel result of Theorem 3 for the case of money supply control of the central bank.

Theorem 4 Suppose Assumptions 1 - 4, 6 and 9 hold. For every $0 \ll$ $\bar{M} \ll+\infty$, there exists a monetary equilibrium with money supply control and fixed transfers $\left\{(\bar{p}, \bar{q}, \bar{r}),\left(\bar{x}^{i}, \bar{m}^{i}, \bar{b}^{i}, \bar{\theta}^{i}\right)_{i \in I},(\bar{M}, \bar{B}, \bar{H})\right\}$.

\subsection{Interpretation and literature}

An intuition for the absence of an indeterminacy statement in Theorem 3 and Theorem 4 can again be given by counting equations and unknowns. As in Section 3.3 for the Ricardian economy, we still have one Walras law in every effective equilibrium. However, unlike the balanced transfer policy, (9)-(11) represent another $S$ equilibrium restrictions in the fixed transfer case, as will be argued in the next paragraph. In total, commodity prices plus the interest rates plus the martingale measure constitute $L(S+1)+(S+1)+(S-1)$ variables which have to be determined. The commodity and money market clearing conditions plus the $S$ restrictions from (9)-(11) minus the single Walras law add up to $L(S+1)+(S+1)+S-1$ equilibrium restrictions. Under interest rate peg, both the money market clearing equations and the interest rates can not be counted. Hence, under interest rate peg and under money supply control, the number of unknowns and restrictions coincides. This is the intuition for why we do not find some degree of indeterminacy here. ${ }^{15}$

The claim of $S$ equilibrium restrictions from (9)-(11) follows both from the fact that the transfers are fixed and from the assumption that the government only trades riskless bonds. The fixed transfer policy always imposes one equilibrium restriction through the government intertemporal equation (18), which is independent of the portfolio set of government. Whether the fixed transfers can add other restrictions depends on the portfolio restrictions the government faces. Our assumption that the government only

\footnotetext{
${ }^{15}$ The same logic as in Footnote 11 can be applied for the monetary equilibrium according to Definition 4. The difference to the argument given in Footnote 11 is that the fixed transfer policy does not imply another $S$ degrees of redundancies in the terminal nodes. Hence, the number of equations now coincides with the number of unknowns.
} 
trades riskless bonds implies that it enters period one with a fixed composition of debt across states. To allow for budget balance, taxes and seigniorage across states must also follow the same composition. This imposes another $S-1$ restrictions to the equilibrium. Hence, there are $S$ total restrictions from the fixed transfer policy and Assumption 4.

To understand the role of Assumption 4 from a broader perspective, suppose that the government has access to $\widehat{J} \leq J$ assets in the economy with $J$ assets. If the government controls the portfolio composition of $\widehat{J}$ assets exogenously, the same logic as in the previous paragraph still applies. Consequently, even if the government controls a fixed portfolio composition other than that of riskless bonds, it seems natural to conjecture that the fixed transfer policy will still add $S$ restrictions to the equilibrium (see Nakajima and Polemarchakis, 2001). But if the government does not actively control its portfolio composition of $\widehat{J}$ assets, the previous logic will not apply. To start with a corner example, suppose that there is a full set of Arrow securities and that the government trades every such security without any restrictions. Then there is only one additional restriction from (18) because of the exogenous transfers, which leads to $S-1$ dimensional indeterminacy even with fixed transfer fiscal policy (see Bloise et al., 2005). More generally, an educated conjecture for the case with $\widehat{J}$ assets would be that (9)-(11) will add $S-\widehat{J}+1$ restrictions to the equilibrium set, leaving the number of indeterminacy as $\widehat{J}-1$. Among these $S-\widehat{J}+1$ restrictions, $S-\widehat{J}$ come from the restrictions of the asset span on the government policy variables, and one comes from the intertemporal restriction (18). Again we emphasize that this is only a conjecture and needs to be verified in a formal study.

The main contributions to the theoretical literature ${ }^{16}$ in economies with non-Ricardian fiscal policies and an active monetary policy are Dubey and Geanakoplos (1992, 2003a, 2003b, 2006). Dubey and Geanakoplos (1992, 2003a) consider a one period model with a cash-in-advance constraint, inside and outside money. Dubey and Geanakoplos (2003b) extend this model to a stochastic economy with incomplete asset markets and a mixed asset structure. In all papers, they show, among several other results, existence of the equilibrium. They do so by using a strategic market game approach. Dubey and Geanakoplos (2006) formally prove generic local uniqueness in the stochastic economy with incomplete asset markets and nominal assets.

We study a similar economy as Dubey and Geanakoplos (2003b, 2006), but to prove existence we basically follow the ideas in Bloise et al. (2005)

\footnotetext{
${ }^{16}$ As opposed to the quite huge macroeconomic literature on the Fiscal Theory of the Price Level.
} 
by introducing a price determination mechanism in the fixed point mapping for every price object. This allows us to establish a unified framework to prove existence of equilibrium in all four cases we consider. In addition, by embedding each equilibrium object into the fixed point mapping, we provide a clear intuition for the mechanism which determines the equilibrium.

Gourdel and Triki (2005) provide a result similar to our Theorem 3 under interest rate peg policy. In addition to the differences mentioned in Section 3.3, there is one more major distinction in this case. While Gourdel and Triki (2005) need strictly positive taxes to establish the existence of a monetary equilibrium, our result also allows for the possibility of zero taxes provided that the economy has sufficiently high gains to trade.

\section{Concluding remarks}

To conclude the paper, we discuss some directions of future research. First, a different timing of transactions can be considered. One possibility is to use the cash-in-advance constraint as introduced by Svensson (1985), where the commodity markets open before the asset markets. This could be a suitable framework to study both the transaction and precautionary demand for money. However, different from our two-period model, the new timing needs an infinite horizon to support money's value. Second, it would be interesting to introduce a Baumol-Tobin structure in which households voluntarily hold money as a store of value even though other interest bearing bonds coexist. Both existence and determinacy in the Baumol-Tobin economy are open and difficult questions. Doing so probably requires more than two periods to enrich the potential transaction patterns. In particular, an infinite horizon model would be of interest. Finally, the model presented here delivers a unified framework for monetary and fiscal policy within a GEI-economy. Therefore, it would be of interest to study the general equilibrium effects of changing monetary policy parameters. Under incomplete financial market the effect can be expected to be real, an important feature for policy analysis. Such an analysis would contribute to the old but fundamental debate about the neutrality of money.

\section{Appendix}

In this appendix we give the proof for the theorems in the main text. The proofs are organized as follows. First, we define an abstract economy. Second, we show the properties of the household and aggregate demand. Then 
we prove the results under different monetary-fiscal policy combinations.

\subsection{An abstract economy}

Define the inverse price level as $c:=\frac{1}{\sum_{l} p_{0 l}+\sum_{s \in S, l} \mu_{s} p_{s l}}$ and the new prices by $\pi_{s l}:=c \mu_{s} p_{s l}$ for all $s \in S$ and $\pi_{0 l}:=c p_{0 l}$. By construction $\pi$ lies in the unit simplex

$$
\Delta:=\left\{\pi \in \mathbb{R}_{+}^{(S+1) L} \mid \sum_{s, l} \pi_{s l}=1\right\} .
$$

Multiply (4) with $c$ and use the no-arbitrage equation $q=\frac{1}{1+r_{0}}(\mu \cdot A)$ to get

$$
\pi_{0} \cdot x_{0}^{i}+\frac{1}{1+r_{0}}\left(\widetilde{b}_{0}^{i}+\mu \cdot A \cdot \widetilde{\theta}^{i}\right)+\widetilde{m}_{0}^{i}=\delta^{i} \widetilde{H}_{0}+\pi_{0} \cdot e_{0}^{i},
$$

where $\widetilde{b}_{0}^{i}:=c b_{0}^{i}, \widetilde{\theta}^{i}:=c \theta^{i}, \widetilde{m}_{0}^{i}:=c m_{0}^{i}$ and $\widetilde{H}_{0}:=c H_{0}$. The cash-in-advance constraint in $t=0$ is

$$
\widetilde{m}_{0}^{i} \geq \pi_{0} \cdot\left(x_{0}^{i}-e_{0}^{i}\right)^{-} .
$$

Multiply (6) by $c \mu$ to get

$$
\pi_{s} \cdot x_{s}^{i}+\frac{\widetilde{b}_{s}^{i}}{1+r_{s}}+\widetilde{m}_{s}^{i}=\mu_{s}\left(\widetilde{b}_{0}^{i}+A_{s} \cdot \widetilde{\theta}^{i}+\widetilde{m}_{0}^{i}-\pi_{0} \cdot \tau_{0}^{i}\right)+\pi_{s} \cdot e_{s}^{i}+\delta^{i} \widetilde{H}_{s}
$$

where $\widetilde{b}_{s}^{i}:=c \mu_{s} b_{s}^{i}, \widetilde{m}_{s}^{i}:=c \mu_{s} m_{s}^{i}$ and $\widetilde{H}_{s}:=c \mu_{s} H_{s}$. The cash-in-advance constraint at state $s \in S$ becomes

$$
\widetilde{m}_{s}^{i} \geq \pi_{s} \cdot\left(x_{s}^{i}-e_{s}^{i}\right)^{-},
$$

and the terminal condition is

$$
\widetilde{b}_{s}^{i}+\widetilde{m}_{s}^{i}-\pi_{s} \cdot \tau_{s}^{i}=0 .
$$

We can now redefine household $i$ 's budget set by

$B^{i}(\pi, \mu, r, \widetilde{H})=\left\{\left(x^{i}, \widetilde{m}^{i}, \widetilde{b}^{i}, \widetilde{\theta}^{i}\right) \in \mathbb{R}_{+}^{(S+1) L} \times \mathbb{R}_{+}^{S+1} \times \mathbb{R}^{S+1} \times \mathbb{R}^{J-1} \mid(20)-(24)\right\}$.

By redefining variables, the intertemporal constraint (17) becomes

$$
\begin{aligned}
& \pi_{0} \cdot x_{0}^{i}+\frac{\pi_{\mathbf{1}} \cdot x_{\mathbf{1}}^{i}}{1+r_{0}}+\frac{r_{0}}{1+r_{0}} \widetilde{m}_{0}^{i}+\frac{1}{1+r_{0}} \frac{r_{\mathbf{1}}}{1+r_{\mathbf{1}}} \cdot \widetilde{m}_{\mathbf{1}}^{i}=\delta^{i}\left(\widetilde{H}_{0}+\frac{\widetilde{H}_{\mathbf{1}} \cdot \mathbf{1}}{1+r_{0}}\right) \\
& +\pi_{0} \cdot\left(e_{0}^{i}-\frac{\tau_{0}^{i}}{1+r_{0}}\right)+\frac{1}{1+r_{0}} \pi_{\mathbf{1}} \cdot\left(e_{\mathbf{1}}^{i}-\frac{1}{1+r_{\mathbf{1}}} \square \tau_{\mathbf{1}}^{i}\right) .
\end{aligned}
$$


The household 1's budget constraint is

$$
B^{1}(\pi, r, \widetilde{H})=\left\{\left(x^{1}, \widetilde{m}^{1}\right) \in \mathbb{R}_{+}^{(S+1) L} \times \mathbb{R}_{+}^{S+1} \mid(21),(23) \text { and (25) hold }\right\} .
$$

It is easy to understand that (9), (10) and (11) are equivalent to (19). With the obvious definitions, equation (19) becomes in the abstract economy

$$
\frac{r_{s}}{1+r_{s}} \widetilde{M}_{s}+\mu_{s} r_{0} \widetilde{M}_{0}+\frac{\pi_{s} \cdot \tau_{s}}{1+r_{s}}+\mu_{s} \pi_{0} \cdot \tau_{0}=\left(1+r_{0}\right) \mu_{s} \widetilde{H}_{0}+\widetilde{H}_{s}, \quad \forall s \in S .
$$

The arguments we used to derive (25) can also be used to derive an intertemporal reformulation of (9), (10) and (11),

$$
\begin{gathered}
\frac{r_{0}}{1+r_{0}} \widetilde{M}_{0}+\frac{1}{1+r_{0}} \frac{r_{\mathbf{1}}}{1+r_{\mathbf{1}}} \cdot \widetilde{M}_{\mathbf{1}}+\frac{\pi_{0} \cdot \tau_{0}}{1+r_{0}} \\
+\frac{1}{1+r_{0}} \pi_{\mathbf{1}} \cdot\left(\frac{1}{1+r_{\mathbf{1}}} \square \tau_{\mathbf{1}}\right) \\
=\widetilde{H}_{0}+\frac{\widetilde{H}_{\mathbf{1}} \cdot \mathbf{1}}{1+r_{0}}
\end{gathered}
$$

A monetary equilibrium in this abstract economy is a vector $\{(\bar{\pi}, \bar{\mu}, \bar{r}, \bar{c})$, $\left.\left(\bar{x}^{i}, \overline{\widetilde{m}}^{i}, \widetilde{\widetilde{b}}^{i}, \overline{\widetilde{\theta}}^{i}\right)_{i \in I},(\overline{\widetilde{M}}, \overline{\widetilde{B}}, \overline{\widetilde{H}})\right\}$ such that markets clear, households optimize, (26) is true, $\bar{c}>0$ and $\bar{\mu} \gg 0$. Such an equilibrium corresponds to a monetary effective equilibrium $\left\{(\bar{p}, \bar{\mu}, \bar{r}),\left(\bar{x}^{i}, \bar{m}^{i}, \bar{b}^{i}, \bar{\theta}^{i}\right)_{i \in I},(\bar{M}, \bar{B}, \bar{H})\right\}$ according to Definition 5. As argued earlier, the latter vector corresponds to a monetary equilibrium according to Definition 4 . In the following proofs, we will therefore concentrate on equilibria in the abstract economy.

\subsection{The household and market demand}

$\mu$ is an element of the $S$-dimensional unit simplex, which we denote with $\Delta^{S-1}$. The extended positive real line is as usual $\overline{\mathbb{R}}_{+}:=\mathbb{R}_{+} \cup\{+\infty\}$. We start by deriving the properties of the budget sets in the following lemma:

Lemma 1 Under Assumptions 1 and 3, the budget sets satisfy the following properties:

(1.1) For $i \geq 2, B^{i}(\pi, \mu, r, \widetilde{H})$ is a non-empty and upper hemi-continuous correspondence for $(\pi, \mu, r, \widetilde{H}) \in \Delta \times \Delta^{S-1} \times \overline{\mathbb{R}}_{+}^{S+1} \times \mathbb{R}_{+}^{S+1}$.

(1.2) For $i \geq 2, B^{i}(\pi, \mu, r, \widetilde{H})$ is compact for $(\pi, \mu, r, \widetilde{H}) \in \operatorname{interior}(\Delta) \times$ interior $\left(\Delta^{S-1}\right) \times \mathbb{R}_{++}^{S+1} \times \mathbb{R}_{+}^{S+1}$. 
(1.3) For $i \geq 2, B^{i}(\pi, \mu, r, \widetilde{H})$ is lower hemi-continuous for $(\pi, \mu, r, \widetilde{H})$ $\in \operatorname{interior}(\Delta) \times \Delta^{S-1} \times \mathbb{R}_{+}^{S+1} \times \mathbb{R}_{+}^{S+1}$.

(1.4) For $i \geq 2$, if $\widetilde{H}_{0}>0$ then $B^{i}(\pi, \mu, r, \widetilde{H})$ is lower hemi-continuous for $(\pi, \mu, r, \widetilde{H}) \in \Delta \times$ interior $\left(\Delta^{S-1}\right) \times \overline{\mathbb{R}}_{+}^{S+1} \times \mathbb{R}_{+}^{S+1}$.

(1.5) For $i \geq 2$, as long as $r_{0}<+\infty, B^{i}(\pi, \mu, r, \widetilde{H})$ is lower hemi-continuous if $(\pi, \mu, r, \widetilde{H}) \in$ interior $(\Delta) \times$ interior $\left(\Delta^{S-1}\right) \times \overline{\mathbb{R}}_{+}^{S+1} \times \mathbb{R}_{+}^{S+1}$.

(1.6) $B^{1}(\pi, r, \widetilde{H})$ is non-empty and upper hemi-continuous for $(\pi, r, \widetilde{H})$ $\in \Delta \times \overline{\mathbb{R}}_{+}^{S+1} \times \mathbb{R}_{+}^{S+1}$.

(1.7) $B^{1}(\pi, r, \widetilde{H})$ is compact for $(\pi, r, \widetilde{H}) \in$ interior $(\Delta) \times \mathbb{R}_{++}^{S+1} \times \mathbb{R}_{+}^{S+1}$.

(1.8) $B^{1}(\pi, r, \widetilde{H})$ is lower hemi-continuous for $(\pi, r, \widetilde{H}) \in \Delta \times \mathbb{R}_{+}^{S+1} \times \mathbb{R}_{+}^{S+1}$.

(1.9) If $\widetilde{H}_{0}>0$ then $B^{1}(\pi, r, \widetilde{H})$ is lower hemi-continuous for $(\pi, r, \widetilde{H})$ $\in \Delta \times \overline{\mathbb{R}}_{+}^{S+1} \times \mathbb{R}_{+}^{S+1}$.

(1.10) As long as $r_{0}<+\infty$ and $\widetilde{H}_{1}>0, B^{1}(\pi, r, \widetilde{H})$ is lower hemi-continuous if $(\pi, r, \widetilde{H}) \in \Delta \times \overline{\mathbb{R}}_{+}^{S+1} \times \mathbb{R}_{+}^{S+1}$.

\section{Proof:}

(1.1) To check non-emptiness, it is sufficient to notice that $\left(x^{i}, \widetilde{m}^{i}, \widetilde{b}^{i}, \widetilde{\theta}^{i}\right)=$ $\left(0, \pi \square e^{i}, 0,0\right)$ satisfies the equations $(20)-(24)$. Upper hemi-continuity is straightforward.

(1.2) Closedness is obvious. To show the boundedness of $B^{i}(\pi, \mu, r, \widetilde{H})$ under $(\pi, \mu, r) \gg 0$, note that an action $\left(x^{i}, \widetilde{m}^{i}, \widetilde{b}^{i}, \widetilde{\theta}^{i}\right)$ in $B^{i}(\pi, \mu, r, \widetilde{H})$ must satisfy $(25)$, which implies $0 \leq\left(x^{i}, \widetilde{m}^{i}\right) \ll+\infty$. From (24) we know that $\widetilde{b}_{s}^{i}>-\infty$ for every $s \in S$. From the standard no-arbitrage argument, we have $-\infty \ll\left(\widetilde{b}_{0}^{i}, \widetilde{\theta}^{i}\right) \ll+\infty$. From (22) this further implies that $\widetilde{b}_{s}^{i}<+\infty$. 
(1.3) To see that there is an interior point, take $\widetilde{\theta}^{i}=0$ and for every $s \in S^{*}$ take $x_{s}^{i}=0, \widetilde{b}_{s}^{i}=-2 \epsilon_{s}^{i}$ and $\widetilde{m}_{s}^{i}=\pi_{s} \cdot e_{s}^{i}+\frac{\epsilon_{s}^{i}}{1+r_{s}}$ with $\epsilon_{s}^{i}>0$. Using Assumption 3, $\pi \gg 0$ and $r \ll+\infty$, it is easy to see that (20) - (24) hold with a strict inequality for all $\epsilon_{s}^{i}$ small enough. Note that this is true even if $\mu_{s}=0$ for some $s \in S$. This sequence shows that the interior of the budget set is nonempty. It is now easy to see that the interior is lower hemi-continuous. Since the closure of a lower hemicontinuous set is again lower hemi-continuous, the result follows.

(1.4) We only need to check that there is an interior point. Change the sequence defined in (1.3) by $\widetilde{b}_{s}^{i}=0$ for every $s \in S^{*}, \widetilde{m}_{0}^{i}=\pi_{0} \cdot e_{0}^{i}+\frac{\delta^{i} \widetilde{H}_{0}}{2}$ and $\widetilde{m}_{s}^{i}=\pi_{s} \cdot e_{s}^{i}+\mu_{s} \frac{\delta^{i} \widetilde{H}_{0}}{4}$ for every $s \in S$ to see that this is true.

(1.5) Again, use $x^{i}=0$ and $\widetilde{\theta}^{i}=0$. In period zero, take $\widetilde{b}_{0}^{i}=-2 \epsilon_{0}^{i}$, $\widetilde{m}_{0}^{i}=\pi_{0} \cdot e_{0}^{i}+\frac{\epsilon_{0}^{i}}{1+r_{0}}$ with $\epsilon_{0}^{i}>0$ and in period one take $\widetilde{b}_{s}^{i}=0$ and $\widetilde{m}_{s}^{i}=\pi_{s} \cdot e_{s}^{i}+\mu_{s} \frac{\widetilde{m}_{0}^{i}-\pi_{0} \cdot \tau_{0}^{i}}{2}$ to see that the interior is nonempty for $\epsilon_{0}^{i}$ small enough.

(1.6) It holds that $\left(x^{1}, \widetilde{m}^{1}\right)=\left(0, \pi \square e^{1}\right)$ is an element of $B^{1}(\pi, r, \widetilde{H})$. Hence, $B^{1}(\pi, r, \widetilde{H})$ is non-empty. The second part is straightforward.

(1.7) This property follows immediately.

(1.8) To see that the interior of $B^{1}(\pi, r, \widetilde{H})$ is nonempty, take $\tilde{m}_{s}^{1}=\pi_{s}$. $e_{s}^{1}+\epsilon_{s}$ for every $s \in S^{*}, x^{1}=0$ and choose all $\epsilon_{s}>0$ small enough. Note that this argument relies on Assumption 3 and $r \ll+\infty$.

(1.9) Under the assumption $\widetilde{H}_{0}>0$, the same sequence as in (1.8) is an interior point for $\epsilon_{s}^{i}$ small enough for every $s \in S^{*}$.

(1.10) Since $\widetilde{H}_{1}>0$ and $r_{0}<+\infty$, the same argument as in (1.8) applies.

The demand correspondence for every consumer type $i \geq 2$ is defined to be

$$
\begin{aligned}
\left(x^{i}, \widetilde{m}^{i}, \widetilde{b}^{i}, \widetilde{\theta}^{i}\right)(\pi, \mu, r, \widetilde{H}):=\left\{\left(x^{i}, \widetilde{m}^{i}, \widetilde{b}^{i}, \widetilde{\theta}^{i}\right) \in B^{i}(\pi, \mu, r, \widetilde{H}) \mid\right. \\
\left.\left(x^{i}, \widetilde{m}^{i}, \widetilde{b}^{i}, \widetilde{\theta}^{i}\right) \in \arg \max u^{i}\left(x^{i}\right)\right\}
\end{aligned}
$$

Let $\varphi^{i}(\pi, \mu, r, \widetilde{H})$ denote the projection of this demand set onto $\left(x^{i}, \widetilde{m}^{i}\right)$, $\varphi_{x}^{i}(\pi, \mu, r, \widetilde{H})$ the projection of the latter onto $x^{i}$ and $\varphi_{\widetilde{m}}^{i}(\pi, \mu, r, \widetilde{H})$ the 
projection onto $\widetilde{m}^{i}$. Household $i=1$ maximizes his utility by choosing $\left(x^{1}, \widetilde{m}^{1}\right)$ being an element of $B^{1}(\pi, r, \widetilde{H})$. The demand correspondence is $\varphi^{1}(\pi, r, \widetilde{H})$ and the projections are defined as above. We summarize the properties of individual demand in the following lemma:

Lemma 2 Under Assumptions 1 - 3, household demand satisfies the following properties:

(2.1) For $i \geq 2$, $\varphi^{i}(\pi, \mu, r, \widetilde{H})$ is non-empty, compact and convex valued for $(\pi, \mu, r, \widetilde{H}) \in \operatorname{interior}(\Delta) \times \operatorname{interior}\left(\Delta^{S-1}\right) \times \mathbb{R}_{++}^{S+1} \times \mathbb{R}_{+}^{S+1}$.

(2.2) For $i \geq 2, \varphi^{i}(\pi, \mu, r, \widetilde{H})$ is upper hemi-continuous under the conditions given in Lemma (1.3), (1.4) or (1.5).

(2.3) $\varphi^{1}(\pi, r, \widetilde{H})$ is non-empty, compact and convex valued for $(\pi, r, \widetilde{H})$ $\in \operatorname{interior}(\Delta) \times \mathbb{R}_{++}^{S+1} \times \mathbb{R}_{+}^{S+1}$.

(2.4) $\varphi^{1}(\pi, r, \widetilde{H})$ is upper hemi-continuous under the conditions given in Lemma (1.8), (1.9) or (1.10).

(2.5) Under the assumption of Lemma (1.8), (1.9) or (1.10),

$$
\inf \left\{\|x\| \mid x \in \varphi_{x}^{1}(\pi, r, \widetilde{H})\right\} \rightarrow+\infty
$$

if $\pi_{s l} \rightarrow 0$ for some $s \in S^{*}$ and $l \in L$.

(2.6) $\forall s \in S^{*}$, if $r_{s}>0$, then $\widetilde{m}_{s}^{i} \leq \pi_{s} \cdot e_{s}^{i}$ for all $\left(\ldots, \widetilde{m}_{s}^{i}, \ldots\right) \in \varphi_{\widetilde{m}}^{i}(\pi, \mu, r, \widetilde{H})$ if $i \geq 2$ and for all $\left(\ldots, \widetilde{m}_{s}^{1}, \ldots\right) \in \varphi_{\widetilde{m}}^{1}(\pi, r, \widetilde{H})$.

(2.7) For every $i \geq 2$, under the conditions of Lemma (1.4) it holds that if $r_{0} \rightarrow+\infty$, then $\widetilde{m}_{0}^{i} \rightarrow 0$ for all $\left(\widetilde{m}_{0}^{i}, \widetilde{m}_{1}^{i} \ldots, \widetilde{m}_{S}^{i}\right) \in \varphi_{\widetilde{m}}^{i}(\pi, \mu, r, \widetilde{H})$. Under the conditions of Lemma (1.9), the same property is true for $i=1$ for every $\left(\widetilde{m}_{0}^{1}, \widetilde{m}_{1}^{1} \ldots, \widetilde{m}_{S}^{1}\right) \in \varphi_{\widetilde{m}}^{1}(\pi, r, \widetilde{H})$.

(2.8) For every $i \geq 2$, under the conditions of Lemma (1.5) it holds that if there is a $s^{\prime} \in S$ with $r_{s^{\prime}} \rightarrow+\infty$, then $\widetilde{m}_{s^{\prime}}^{i} \rightarrow 0$ for all $\left(\widetilde{m}_{0}^{i}, \ldots, \widetilde{m}_{s^{\prime}}^{i}, \ldots, \widetilde{m}_{S}^{i}\right) \in$ $\varphi_{\widetilde{m}}^{i}(\pi, \mu, r, \widetilde{H})$. Under the conditions of Lemma (1.10), the same property is true for $i=1$ for every $\left(\widetilde{m}_{0}^{1}, \widetilde{m}_{1}^{1} \ldots, \widetilde{m}_{S}^{1}\right) \in \varphi_{\widetilde{m}}^{1}(\pi, r, \widetilde{H})$. 
Proof: Parts (2.1) - (2.5) follow from standard arguments using the results from Lemma 1. Since money is dominated as a store of value for a strictly positive interest rate, $\widetilde{m}_{s}^{i}=\pi_{s} \cdot\left(x_{s}^{i}-e_{s}^{i}\right)^{-}, \forall s \in S^{*}, \forall i \in I$. This implies (2.6). Concerning (2.7), we first argue for $i \geq 2$. For $r_{0} \rightarrow+\infty$ we argue that the sequence of best responses converges to a $\left(x^{i}, \widetilde{m}^{i}, \widetilde{b}^{i}, \widetilde{\theta}^{i}\right)$ such that $\widetilde{m}_{0}^{i}=0$. From Lemma (2.2), the demand set is upper hemi-continuous along this sequence. We will argue that if $\widetilde{m}_{0}^{i}>0$ in the limit, then the household can increase his utility. Since the cash-in-advance constraint binds in the case of positive interest rates, $\widetilde{m}_{0}^{i}>0$ implies that he sells something of his endowment. If he deviates by selling nothing and consuming what he sold before, his utility increases. The revenue which he loses in period one from not selling the endowment in period zero can be taken from buying costless bonds. This deviation implies that $\widetilde{m}_{0}^{i}>0$ cannot be the best response in the limit. For household $i=1$, the same property is true. In fact, by optimality, $\frac{r_{s}}{1+r_{s}} \widetilde{m}_{s}^{i}=\frac{r_{s}}{1+r_{s}} \pi_{s} \cdot\left(x_{s}^{i}-e_{s}^{i}\right)^{-}$. Using this in (25) implies

$$
\begin{aligned}
\pi_{0} \cdot\left(x_{0}^{1}-e_{0}^{1}\right)^{+}+\frac{\pi_{\mathbf{1}} \cdot\left(x_{\mathbf{1}}^{1}-e_{\mathbf{1}}^{1}\right)^{+}}{1+r_{0}}=\delta^{1}\left(\widetilde{H}_{0}+\frac{\widetilde{H}_{\mathbf{1}} \cdot \mathbf{1}}{1+r_{0}}\right) \\
\quad+\frac{\pi_{0} \cdot\left(\left(x_{0}^{1}-e_{0}^{1}\right)^{-}-\tau_{0}^{1}\right)}{1+r_{0}}+\frac{1}{1+r_{0}} \pi_{\mathbf{1}} \cdot\left(\left(\left(x_{\mathbf{1}}^{1}-e_{\mathbf{1}}^{1}\right)^{-}-\tau_{1}^{1}\right) \square \frac{1}{1+r_{\mathbf{1}}}\right) .
\end{aligned}
$$

This equation reveals that household one earns zero from selling his endowments in $t=0$. Maximization thus implies selling no endowments. From the cash-in-advance it follows that money demand is zero. Part (2.8) follows from the same logic as part (2.7).

Define the market demand correspondence of the commodity and money as

$$
Z(\pi, \mu, r, \widetilde{H}):=\varphi^{1}(\pi, r, \widetilde{H})+\sum_{i \geq 2} \varphi^{i}(\pi, \mu, r, \widetilde{H})
$$

and the projections of this set onto commodity and money spaces by $Z_{x}(\pi, \mu, r, \widetilde{H})$ and $Z_{\widetilde{m}}(\pi, \mu, r, \widetilde{H})$, respectively.

Lemma 3 Under Assumptions 1 - 3, Z $(\pi, \mu, r, \widetilde{H})$ satisfies the following properties:

$$
\begin{aligned}
& Z(\pi, \mu, r, \tilde{H}) \text { is non-empty, compact and convex-valued for }(\pi, \mu, r, \widetilde{H}) \\
& \in \text { interior }(\Delta) \times \text { interior }\left(\Delta^{S-1}\right) \times \mathbb{R}_{++}^{S+1} \times \mathbb{R}_{+}^{S+1}
\end{aligned}
$$


(3.2) $Z(\pi, \mu, r, \widetilde{H})$ is upper hemi-continuous for $(\pi, \mu, r, \widetilde{H}) \in \operatorname{interior}(\Delta) \times$ $\Delta^{S-1} \times \mathbb{R}_{+}^{S+1} \times \mathbb{R}_{+}^{S+1}$.

(3.3) If $\left(z_{x}, z_{\widetilde{m}}\right) \in Z(\pi, \mu, r, \widetilde{H})$ and if (27) holds, then

$$
\begin{gathered}
\left(1+r_{0}\right) \pi_{0} \cdot\left(z_{x_{0}}-\sum_{i} e_{0}^{i}\right)+\pi_{\mathbf{1}} \cdot\left(z_{x_{\mathbf{1}}}-\sum_{i} e_{\mathbf{1}}^{i}\right) \\
+r_{0}\left(z_{\widetilde{m}_{0}}-\widetilde{M}_{0}\right)+\frac{r_{\mathbf{1}}}{1+r_{\mathbf{1}}} \cdot\left(z_{\widetilde{m}_{\mathbf{1}}}-\widetilde{M}_{\mathbf{1}}\right)=0 .
\end{gathered}
$$

(3.4) If $z_{\widetilde{m}} \in Z_{\widetilde{m}}(\pi, \mu, r, \widetilde{H})$ then $z_{\widetilde{m}_{s}} \leq \max _{s, l} \sum_{i} e_{s l}^{i}$ for $r \gg 0$ and all $s \in S^{*}$.

Proof: Lemma (3.1), (3.2) and (3.4) follow directly from individual demand (Lemma 2). Lemma (3.3) follows from adding up (25) over $i \in I$ and using (27).

\subsection{Proof of Theorem 1}

We fix the martingale measure $\mu \gg 0$ and the inverse price level $c>0$ at the outset. The transfers are determined endogenously according to the balanced transfer rule.

\subsubsection{Preliminary definitions}

From Assumption 2, we can define a government transfer function $\widetilde{H}(\pi, r, \widetilde{M}):=$ $\left(\widetilde{H}_{0}, \widetilde{H}_{1}, \ldots, \widetilde{H}_{S}\right)(\pi, r, \widetilde{M})$, where

$$
\widetilde{H}_{s}(\pi, r, \widetilde{M}):=\frac{r_{s}}{1+r_{s}} \widetilde{M}_{s}+\frac{\pi_{s} \cdot \tau_{s}}{1+r_{s}}, \forall s \in S^{*}
$$

We slightly abuse the notation by denoting both the function and the image with $\widetilde{H}$. By construction, $\widetilde{H}_{s} \geq 0, \forall s \in S^{*}$. Obviously, $\widetilde{H}(\pi, r, \widetilde{M})$ is a bounded and continuous function for $(\pi, r, \widetilde{M}) \in \Delta \times \mathbb{R}_{+}^{S+1} \times \mathbb{R}_{+}^{S+1}$.

\subsubsection{Construction of a fixed point mapping}

To make the proof compatible with a zero interest rate, we start by defining the modified interest rate process $r^{n}:=\left(r_{s}^{n}\right)_{s \in S^{*}}$ by

$$
r_{s}^{n}=\left\{\begin{array}{cl}
r_{s} & \text { if } r_{s}>0 \\
\frac{1}{n} & \text { if } r_{s}=0
\end{array}\right.
$$


For $n>(S+1) L$ define

$$
\Delta^{n}:=\left\{\pi \in \Delta \mid \pi_{s l} \geq \frac{1}{n}\right\}
$$

It is easy to see that $\bigcup_{n>(S+1) L}^{\infty} \Delta^{n}=\operatorname{interior}(\Delta)$. Let $K_{\widetilde{m}}$ be a compact and convex space such that $K_{\widetilde{m}} \supseteq Z_{\widetilde{m}}^{n}(\pi, \widetilde{H})$ for all $\pi \in \Delta$ and $\widetilde{H} \in$ $\mathbb{R}_{+}^{S+1}$, where $Z_{\widetilde{m}}^{n}(\pi, \widetilde{H}):=Z_{\widetilde{m}}\left(\pi, \mu, r^{n}, \widetilde{H}\right)$. Since $r^{n} \gg 0$ for all finite $n$, such a compact set exists by Lemma (3.4). ${ }^{17}$ Define a compact and convex set $K_{\widetilde{H}}$ such that $K_{\widetilde{H}} \supseteq \widetilde{H}^{n}(\pi, \widetilde{M})$ for all $\pi \in \Delta$ and $\widetilde{M} \in K_{\widetilde{m}}$, where $\widetilde{H}^{n}(\pi, \widetilde{M}):=\widetilde{H}\left(\pi, r^{n}, \widetilde{M}\right)$. Since $\widetilde{H}$ is a bounded function and $\widetilde{M} \in K_{\widetilde{m}}$, such a set $K_{\widetilde{H}}$ exists. Further define $Z_{x}^{n}(\pi, \widetilde{H}):=Z_{x}\left(\pi, \mu, r^{n}, \widetilde{H}\right)$ and a compact and convex set $K_{x}^{n}$ such that $K_{x}^{n} \supseteq Z_{x}^{n}(\pi, \widetilde{H})$ for all $\pi \in \Delta^{n}$ and $\widetilde{H} \in K_{\widetilde{H}}$. Denote the product set with $K^{n}:=K_{x}^{n} \times K_{\widetilde{m}}$. Note that only $K_{x}^{n}$ depends on $n$. Finally, define the mapping

$$
f^{n}: \Delta^{n} \times K_{\widetilde{m}} \times K_{\widetilde{H}} \times K^{n} \rightrightarrows \Delta^{n} \times K_{\widetilde{m}} \times K_{\widetilde{H}} \times K^{n}
$$

by

$$
(\pi, \widetilde{M}, \widetilde{H}, z) \stackrel{f^{n}}{\mapsto}\left(f_{\pi}^{n}, f_{\widetilde{M}}^{n}, f_{\widetilde{H}}^{n}, f_{z}^{n}\right),
$$

where

$$
\begin{aligned}
f_{\pi}^{n}(\pi, \widetilde{M}, \widetilde{H}, z) & :=\underset{\left\{\pi \in \Delta^{n}\right\}}{\arg \max }\left\{\left(1+r_{0}\right) \pi_{0} \cdot\left(z_{x_{0}}-\sum_{i} e_{0}^{i}\right)+\pi_{\mathbf{1}} \cdot\left(z_{x_{\mathbf{1}}}-\sum_{i} e_{\mathbf{1}}^{i}\right)\right\}, \\
f_{\widetilde{M}}^{n}(\pi, \widetilde{M}, \widetilde{H}, z) & :=z_{\widetilde{m}} \\
f_{\widetilde{H}}^{n}(\pi, \widetilde{M}, \widetilde{H}, z) & :=\widetilde{H}^{n}(\pi, \widetilde{M}), \\
f_{z}^{n}(\pi, \widetilde{M}, \widetilde{H}, z) & :=Z^{n}(\pi, \widetilde{H}) .
\end{aligned}
$$

The first mapping is the price player's objective function, the second mapping says that the government accommodates money demand, the third mapping is the government transfer function and the last mapping is the market demand.

From Lemma 3 we infer that $f^{n}(\pi, \widetilde{M}, \widetilde{H}, z)$ is a non-empty, compact, convex-valued and upper hemi-continuous correspondence. Kakutani Fixed Point Theorem establishes the existence of a fixed point $\left(\pi^{* n}, \widetilde{M}^{* n}, \widetilde{H}^{* n}, z^{* n}\right)$.

\footnotetext{
${ }^{17}$ Even though $r$ depends on $n$, the set $K_{\widetilde{m}}$ does not depend on $n$ by Lemma (3.4).
} 


\subsubsection{The limit of the fixed points is an equilibrium}

Since $\left(\pi^{* n}, \widetilde{M}^{* n}, \widetilde{H}^{* n}, z^{* n}\right)$ is bounded for each $n$, we can choose a subsequence having a limit $\left(\pi^{*}, \widetilde{M^{*}}, \widetilde{H}^{*}, z^{*}\right) . \pi^{*}$ is trivially bounded. By Lemma $(3.4), z_{\widetilde{m}}^{*}$ is also finite since $z_{\widetilde{m}}^{n}$ is bounded above by the aggregate endowment for all $n$. By construction, $\widetilde{M}^{*}=z_{\widetilde{m}}^{*}$. Since $\widetilde{H}(\pi, \widetilde{M})$ is continuous, $\widetilde{H}^{*}=\widetilde{H}\left(\pi^{*}, \widetilde{M}^{*}\right)$. This implies that $\widetilde{H}^{*}$ is finite. It only remains to show that $z_{x}^{*}=\sum_{i} e^{i}$ and $z_{x}^{*} \in Z_{x}\left(\pi^{*}, \widetilde{H}^{*}\right)$.

It follows from $\widetilde{M}^{*}=z_{\widetilde{m}}^{*}$ and Lemma (3.3) that for all $n$

$$
\left(1+r_{0}\right) \pi_{0}^{* n} \cdot\left(z_{x_{0}}^{* n}-\sum_{i} e_{0}^{i}\right)+\pi_{\mathbf{1}}^{* n} \cdot\left(z_{x_{\mathbf{1}}}^{* n}-\sum_{i} e_{\mathbf{1}}^{i}\right)=0
$$

which implies

$$
\left(1+r_{0}\right) \pi_{0}^{*} \cdot\left(z_{x_{0}}^{*}-\sum_{i} e_{0}^{i}\right)+\pi_{\mathbf{1}}^{*} \cdot\left(z_{x_{\mathbf{1}}}^{*}-\sum_{i} e_{\mathbf{1}}^{i}\right)=0
$$

in the limit. Consequently, we have $z_{x}^{*} \leq \sum_{i} e^{i}$, and $z_{x}^{*}=\sum_{i} e^{i}$ if $\pi^{*} \gg 0$. However, from Lemma (2.5) we know that household one's demand goes to infinity if some $\pi_{s l}^{*} \rightarrow 0$. Since aggregate excess demand is bounded below, we get that $\left\|z_{x}^{*}\right\| \rightarrow+\infty$ if some $\pi_{s l}^{*} \rightarrow 0$. Therefore, $z_{x}^{*} \leq \sum_{i} e^{i}$ implies that $\pi^{*} \gg 0$ and $z_{x}^{*}=\sum_{i} e^{i}$. Since $Z_{x}(\pi, \widetilde{H})$ is upper hemi-continuous for $\pi \gg 0$, we know that $z_{x}^{*} \in Z_{x}\left(\pi^{*}, \widetilde{H}^{*}\right)$.

It is straightforward to see that the vector $\left(\pi^{*}, \widetilde{M}^{*}, \widetilde{H}^{*}, z^{*}\right)$ corresponds to a monetary equilibrium in the abstract economy under interest rate peg with balanced transfers.

\subsection{Proof of Theorem 2}

Similar to the proof of Theorem 1, we fix an arbitrary inverse price level $c>0$ and an arbitrary martingale measure $\mu \gg 0$.

\subsubsection{Preliminary definitions}

In the abstract economy, the money supply vector is $\widetilde{M}=c \cdot\left(\bar{M}_{0},\left(\mu_{s} \bar{M}_{s}\right)_{s \in S}\right)$ $\gg 0$. Define the transfers $\widetilde{H}(\pi, r, \widetilde{M})$ to individuals as in Section 6.3. Since 
$\widetilde{M}$ is fixed here, we write $\widetilde{H}(\pi, r)$.

\subsubsection{Construction of a fixed point mapping}

As before, we define $\Delta^{n}:=\left\{\pi \in \Delta \mid \pi_{s l} \geq \frac{1}{n}\right\}$ for $n>(S+1) L$. Define the set $\Omega^{n}:=\left[\frac{1}{n}, n\right]^{S+1}$ carrying the interest rates $r$. Let $K_{\widetilde{m}}$ be a compact and convex space such that $K_{\widetilde{m}} \supseteq Z_{\widetilde{m}}(\pi, r, \widetilde{H})$ for all $\pi \in \Delta^{n}, r \in \Omega^{n}$ and $\widetilde{H} \in \mathbb{R}_{+}^{S+1}$. Define a set $K_{\widetilde{H}}$ such that $K_{\widetilde{H}} \supseteq \widetilde{H}(\pi, r)$ for all $\pi \in \Delta^{n}$ and $r \in \Omega^{n}$. Define the compact and convex set $K_{x}^{n}$ such that $K_{x}^{n} \supseteq Z_{x}^{n}(\pi, r, \widetilde{H})$ for all $\pi \in \Delta^{n}, r \in \Omega^{n}$ and $\widetilde{H} \in \mathbb{R}_{+}^{S+1}$. The product set is $K^{n}:=K_{x}^{n} \times K_{\widetilde{m}}$. As before, define the mapping

$$
f^{n}: \Delta^{n} \times \Omega^{n} \times K_{\widetilde{H}} \times K^{n} \rightrightarrows \Delta^{n} \times \Omega^{n} \times K_{\widetilde{H}} \times K^{n}
$$

by

$$
(\pi, r, \widetilde{H}, z) \stackrel{f^{n}}{\mapsto}\left(f_{\pi}^{n}, f_{r}^{n}, f_{\widetilde{H}}^{n}, f_{z}^{n}\right)
$$

where

$$
\begin{aligned}
f_{\pi}^{n}(\pi, r, \widetilde{H}, z) & :=\underset{\left\{\pi \in \Delta^{n}\right\}}{\arg \max }\left\{\left(1+r_{0}\right) \pi_{0} \cdot\left(z_{x_{0}}-\sum_{i} e_{0}^{i}\right)+\pi_{\mathbf{1}} \cdot\left(z_{x_{\mathbf{1}}}-\sum_{i} e_{\mathbf{1}}^{i}\right)\right\}, \\
f_{r}^{n}(\pi, r, \widetilde{H}, z) & :=\underset{r \in \Omega^{n}}{\arg \max }\left\{r_{0}\left(z_{\widetilde{m}_{0}}-\widetilde{M}_{0}\right)+\frac{r_{\mathbf{1}}}{1+r_{\mathbf{1}}} \cdot\left(z_{\widetilde{m}_{\mathbf{1}}}-\widetilde{M}_{\mathbf{1}}\right)\right\}, \\
f_{\widetilde{H}}^{n}(\pi, r, \widetilde{H}, z) & :=\widetilde{H}(\pi, r), \\
f_{z}^{n}(\pi, r, \widetilde{H}, z) & :=Z(\pi, r, \widetilde{H}) .
\end{aligned}
$$

Again, all these mappings satisfy the assumptions required to apply Kakutani's Theorem, implying the existence of a fixed point $\left(\pi^{* n}, r^{* n}, \widetilde{H}^{* n}, z^{* n}\right)$.

\subsubsection{The limit of the fixed points is an equilibrium}

Since $\left(\pi^{* n}, r^{* n}, \widetilde{H}^{* n}, z^{* n}\right)$ is bounded for each $n$, we can choose a subsequence having a limit $\left(\pi^{*}, r^{*}, \widetilde{H}^{*}, z^{*}\right)$. For every such subsequence, $\left(\pi^{*}, \widetilde{H}^{*}, z_{\widetilde{m}}^{*}\right) \ll$ $+\infty$. Since $\widetilde{H}(\pi, r)$ is continuous, we know that $\widetilde{H}^{*}=\widetilde{H}\left(\pi^{*}, r^{*}\right)$. Hence it remains to show that markets clear and $\left(r^{*}, z_{x}^{*}\right) \ll+\infty$.

Claim 1: In period zero, the money market clears and $r_{0}^{*}<+\infty$. To see this, we argue in three steps. 
Step 1: We prove that $z_{\widetilde{m}_{0}}^{*} \leq \widetilde{M}_{0}$. Suppose not, i.e., $z_{\widetilde{m}_{0}}^{*}>\widetilde{M}_{0}$. From the construction of $f_{r}^{n}$, we must have $r_{0}^{*}=+\infty$. Then it follows from the definition of $\widetilde{H}\left(\pi^{*}, r^{*}\right)$ that $\widetilde{H}_{0}^{*}$ is strictly positive. Lemma $(2.7)$ implies that $z_{\widetilde{m}_{0}}^{*}=0$, a contradiction to $z_{\widetilde{m}_{0}}^{*}>\widetilde{M}_{0}^{*}>0$. Hence $z_{\widetilde{m}_{0}}^{*} \leq \widetilde{M}_{0}$.

Step 2: We prove that $z_{\widetilde{m}_{0}}^{*}=\widetilde{M}_{0}$ if $r_{0}^{*}>0$. Since the construction of $f_{r}^{n}$ implies $r_{0}^{*}\left(z_{\widetilde{m}_{0}}^{*}-\widetilde{M}_{0}\right) \geq 0$ and $z_{\widetilde{m}_{0}}^{*} \leq \widetilde{M}_{0}$ implies $r_{0}^{*}\left(z_{\widetilde{m}_{0}}^{*}-\widetilde{M}_{0}\right) \leq 0$, we must have $r_{0}^{*}\left(z_{\widetilde{m}_{0}}^{*}-\widetilde{M}_{0}\right)=0$. Therefore, $r_{0}^{*}>0$ implies $z_{\widetilde{m}_{0}}^{*}-\widetilde{M}_{0}=0$, which means the money market clearing in period zero (with free disposal) in the limit.

Step 3: We prove $r_{0}^{*}<+\infty$. Suppose that $r_{0}^{*}=+\infty$. From the first step, we know that $z_{\widetilde{m}_{0}}^{*}=0$; from the second step, we know $z_{\widetilde{m}_{0}}^{*}=\widetilde{M}_{0}$. These two facts imply $\widetilde{M}_{0}=0$, a contradiction.

Claim 2: For every $s \in S$, the money market in state $s$ clears and $r_{s}^{*}<+\infty$. To see this, we argue in several steps.

Step 1: We show that $z_{x_{0}}^{*} \leq \sum_{i} e_{0}^{i}$ and $z_{x_{\mathbf{1}}}^{*} \leq \sum_{i} e_{\mathbf{1}}^{i}$. Indeed, using the definition of $\widetilde{H}(\pi, r)$ and Lemma $(3.3)$, it follows $\left(1+r_{0}^{*}\right) \pi_{0}^{*} \cdot\left(z_{x_{0}}^{*}-\sum_{i} e_{0}^{i}\right)+$ $\pi_{\mathbf{1}}^{*} \cdot\left(z_{x_{1}}^{*}-\sum_{i} e_{\mathbf{1}}^{i}\right)+r_{0}^{*}\left(z_{\widetilde{m}_{0}}^{*}-\widetilde{M}_{0}\right)+\frac{r_{1}^{*}}{1+r_{1}^{*}} \cdot\left(z_{\widetilde{m}_{\mathbf{1}}}^{*}-\widetilde{M}_{\mathbf{1}}\right)=0$. In Claim 1 we established $r_{0}^{*}\left(z_{\widetilde{m}_{0}}^{*}-\widetilde{M}_{0}\right)=0$. In addition, the definition of $f_{r}^{n}$ implies that $\frac{r_{1}^{*}}{1+r_{1}^{*}} \cdot\left(z_{\widetilde{m}_{1}}^{*}-\widetilde{M}_{1}\right) \geq 0$. From this it follows that $\left(1+r_{0}^{*}\right) \pi_{0}^{*} \cdot\left(z_{x_{0}}^{*}-\sum_{i} e_{0}^{i}\right)+$ $\pi_{1}^{*} \cdot\left(z_{x_{1}}^{*}-\sum_{i} e_{1}^{i}\right) \leq 0$. From the definition of $f_{\pi}^{n}$ we get $z_{x_{0}}^{*} \leq \sum_{i} e_{0}^{i}$ and $z_{x_{1}}^{*} \leq \sum_{i} e_{1}^{i}$.

Step 2: The conditions of Lemma (2.5) apply. To see this, we argue that either the conditions of Lemma (1.8) or the conditions of Lemma (1.10) are satisfied. In fact, If $r_{1}^{*} \ll+\infty$, then the conditions of Lemma (1.8) apply trivially. Alternatively, if there is some $s \in S$ with $r_{s}^{*}=+\infty$, then it follows from the definition of $\widetilde{H}(\pi, r)$ that $\widetilde{H}_{1}^{*}>0$. Then the conditions of Lemma (1.10) hold.

Step 3: We show that $\pi^{*} \gg 0$. In fact, we saw in the previous step that Lemma (2.5) can be applied. So if there is an $s \in S^{*}$ and an $l \in L$ such that $\pi_{s l}^{*}=0$, then Lemma (2.5) implies a contradiction to Step 1.

Step 4: Since $r_{0}^{*}<+\infty$ and $\pi^{*} \gg 0$, Lemma (2.8) applies. With this in mind, it is easy to see that the Steps 1-3 of Claim 1 apply. Hence Claim 2 follows.

From money market clearing it follows now by the same arguments as in the proof of Theorem 1 that $z_{x}^{*}=\sum_{i} e^{i}$. From $\pi^{*} \gg 0$ and the upper hemi-continuity of the demand, $z^{*} \in Z\left(\pi^{*}, r^{*}, \widetilde{H}^{*}\right)$. 
Finally, $\left(\pi^{*}, r^{*}, \widetilde{H}^{*}, z^{*}\right)$ corresponds to a monetary equilibrium in the abstract economy with money supply control and balanced transfers.

\subsection{Proof of Theorem 3}

With fixed nominal transfers we just introduce a transfer mapping which transforms the original transfers into discounted real transfers. In addition, we now determine $c$ and $\mu$ endogenously in the fixed point.

\subsubsection{Preliminary definitions}

Define an augmented taxation $\tau^{n} \in \mathbb{R}_{+}^{(S+1) L}$ as

$$
\tau_{s}^{n}=\left\{\begin{array}{cc}
\tau_{s} & \text { if } \tau_{s}>0 \\
\left(\frac{1}{n}, 0, . ., 0\right) \in \mathbb{R}_{+}^{L} & \text { if } \tau_{s}=0
\end{array}\right.
$$

where $n \in \mathbb{N}$ and $n>\min _{s}\left(\frac{1}{e_{s 1}^{1}}\right)$. Without loss of generality, if $\tau_{s}=0$ we can assign the taxation to household 1, i.e., $\tau_{s}^{1 n}=\left(\frac{1}{n}, 0, . ., 0\right)$ and hence $\tau_{s}^{i n}=0$ for all $i \neq 1$. It is easy to see that household 1 will have a non-empty budget set for all $n>\min _{s}\left(\frac{1}{e_{s 1}^{1}}\right)$.

Define a government transfer function $\widetilde{H}(c, \mu):=\left(\widetilde{H}_{0}, \widetilde{H}_{1}, \ldots, \widetilde{H}_{S}\right)(c, \mu)$ by

$$
\widetilde{H}_{0}(c, \mu):=c \bar{H}_{0} \text { and } \widetilde{H}_{s}(c, \mu):=c \mu_{s} \bar{H}_{s}, \forall s \in S .
$$

This function is obviously a bounded and continuous function for finite $c$. Next, define an inverse price level function $c^{n}$ by

$$
c^{n}\left(\pi, z_{\widetilde{m}}, \mu ; r\right):=\frac{\frac{r_{0}}{1+r_{0}} z_{\widetilde{m}_{0}}+\frac{1}{1+r_{0}} \frac{r_{\mathbf{1}}}{1+r_{\mathbf{1}}} \cdot z_{\widetilde{m}_{\mathbf{1}}}+\frac{\pi_{0} \tau_{0}^{n}}{1+r_{0}}+\frac{1}{1+r_{0}} \pi_{\mathbf{1}} \cdot\left(\frac{1}{1+r_{\mathbf{1}}} \square \tau_{\mathbf{1}}^{n}\right)}{\bar{H}_{0}+\sum_{s \in S} \frac{\mu_{s}}{1+r_{0}} \bar{H}_{s}},
$$

and use the shortcut $c^{n}\left(\pi, z_{\widetilde{m}}, \mu\right):=c^{n}\left(\pi, z_{\widetilde{m}}, \mu ; r\right)$. This is a bounded and continuous function of $\left(\pi, z_{\widetilde{m}}, \mu\right)$ for each $n$ as long as $\bar{H}_{0}>0$ and $z_{\widetilde{m}} \ll+\infty$. Under the latter condition, define the bounded and continuous martingalemeasure function $\mu^{n}\left(\pi, z_{\widetilde{m}}, c, \mu\right):=\left(\mu_{1}^{n}, \ldots, \mu_{S}^{n}\right)\left(\pi, c, \mu, z_{\widetilde{m}} ; r\right)$ by

$$
\mu_{s}^{n}\left(\pi, c, \mu, z_{\widetilde{m}} ; r\right):=\frac{\frac{r_{s}}{1+r_{s}} z_{\widetilde{m}_{s}}+\frac{1}{1+r_{s}} \pi_{s} \cdot \tau_{s}^{n}+\mu_{s} c\left(\max _{s^{\prime} \in S} \bar{H}_{s^{\prime}}-\bar{H}_{s}\right)}{\frac{r_{\mathbf{1}}}{1+r_{\mathbf{1}}} \cdot z_{\widetilde{m}_{\mathbf{1}}}+\pi_{\mathbf{1}} \cdot\left(\frac{1}{1+r_{\mathbf{1}}} \square \tau_{\mathbf{1}}\right)+c \sum_{\sigma \in S} \mu_{\sigma}\left(\max _{s^{\prime} \in S} \bar{H}_{s^{\prime}}-\bar{H}_{\sigma}\right)} .
$$

For fixed $r$, we just write $\mu_{s}^{n}\left(\pi, c, \mu, z_{\widetilde{m}}\right)$. By the construction of $\tau^{n}$, as long as $\pi \gg 0$ we have $c^{n}>0, \mu_{s}^{n}>0$ and $\sum_{s=1}^{S} \mu_{s}^{n}=1$ for all finite $n$. 


\subsubsection{Construction of a fixed point mapping}

Denote aggregate demand with $Z^{n}(\pi, \mu, \widetilde{H}):=Z\left(\pi, r, \mu, \widetilde{H}, \tau^{n}\right)$. Lemma (3.4) allows us to define the compact and convex set $K_{\widetilde{m}}$ such that $K_{\widetilde{m}} \supseteq$ $Z_{\widetilde{m}}^{n}(\pi, \mu, \widetilde{H})$ for all $\pi \in \Delta, \mu \in \Delta^{S-1}$ and $\widetilde{H} \in \mathbb{R}_{+}^{S+1}$. Notice that $K_{\widetilde{m}}$ does not depend on $n$. As argued above, for positive $\bar{H}_{0}$ we can define a compact and convex set $K_{c}^{n}$ such that $K_{c}^{n} \supseteq c^{n}\left(\pi, z_{\tilde{m}}, \mu\right)$ for all $\pi \in \Delta$, $z_{\widetilde{m}} \in K_{\widetilde{m}}$ and $\mu \in \Delta^{S-1} \cdot \mu^{n}\left(\pi, c, \mu, z_{\widetilde{m}}\right)$ lies in a compact and convex set $K_{\mu}^{n} \subset \operatorname{interior}\left(\Delta^{S-1}\right)$ for $\pi \in \Delta^{n}, z_{\widetilde{m}} \in K_{\widetilde{m}}$ and $c \in K_{c}^{n}$. Introduce the set $K_{\widetilde{H}}^{n}$ such that $K_{\widetilde{H}}^{n} \supseteq \widetilde{H}(c, \mu)$ for all $c \in K_{c}^{n}$ and $\mu \in K_{\mu}^{n}$. This set can be chosen to be compact and convex for every $n$ since $c \in K_{c}^{n}$. Further define $K_{x}^{n}$ such that $K_{x}^{n} \supseteq Z_{x}^{n}(\pi, \mu, \widetilde{H})$ for all $\pi \in \Delta^{n}, \mu \in K_{\mu}^{n}$ and $\widetilde{H} \in K_{\widetilde{H}}^{n}$. Finally, $K^{n}:=K_{x}^{n} \times K_{\widetilde{m}}$. Define the mapping

$$
f^{n}: \Delta^{n} \times K_{c}^{n} \times K_{\mu}^{n} \times K_{\widetilde{H}}^{n} \times K^{n} \rightrightarrows \Delta^{n} \times K_{c}^{n} \times K_{\mu}^{n} \times K_{\widetilde{H}}^{n} \times K^{n}
$$

by

$$
(\pi, c, \mu, \widetilde{H}, z) \stackrel{f^{n}}{\mapsto}\left(f_{\pi}^{n}, f_{c}^{n}, f_{\mu}^{n}, f_{\widetilde{H}}^{n}, f_{z}^{n}\right)
$$

where

$$
\begin{aligned}
f_{\pi}^{n}(\pi, c, \mu, \widetilde{H}, z) & :=\underset{\left\{\pi \in \Delta^{n}\right\}}{\arg \max }\left\{\left(1+r_{0}\right) \pi_{0} \cdot\left(z_{x_{0}}-\sum_{i} e_{0}^{i}\right)+\pi_{\mathbf{1}} \cdot\left(z_{x_{\mathbf{1}}}-\sum_{i} e_{\mathbf{1}}^{i}\right)\right\} \\
f_{c}^{n}(\pi, c, \mu, \widetilde{H}, z) & :=c^{n}\left(\pi, z_{\widetilde{m}}, \mu\right) \\
f_{\mu}^{n}(\pi, c, \mu, \widetilde{H}, z) & :=\mu^{n}\left(\pi, c, \mu, z_{\widetilde{m})}\right. \\
f_{\widetilde{H}}^{n}(\pi, c, \mu, \widetilde{H}, z) & :=\widetilde{H}(c, \mu) \\
f_{z}^{n}(\pi, c, \mu, \widetilde{H}, z) & :=Z^{n}(\pi, \mu, \widetilde{H}) .
\end{aligned}
$$

$f^{n}(\pi, c, \mu, \widetilde{H}, z)$ is a non-empty, compact, convex-valued and upper hemicontinuous correspondence. Kakutani fixed point theorem establishes the existence of a fixed point $\left(\pi^{* n}, c^{* n}, \mu^{* n}, \widetilde{H}^{* n}, z^{* n}\right)$.

Note that the money market is always cleared since the central bank accommodates money demand. From the construction of $c^{n}\left(\pi, z_{\widetilde{m}}, \mu\right)$, in the fixed point the equation (27) holds, i.e.,

$r_{0} \widetilde{M}_{0}^{* n}+\frac{r_{\mathbf{1}}}{1+r_{\mathbf{1}}} \cdot \widetilde{M}_{\mathbf{1}}^{* n}+\pi_{0}^{* n} \cdot \tau_{0}^{n}+\pi_{\mathbf{1}}^{* n} \cdot\left(\frac{1}{1+r_{\mathbf{1}}} \square \tau_{\mathbf{1}}^{n}\right)=\widetilde{H}_{0}^{* n}\left(1+r_{0}\right)+\widetilde{H}_{\mathbf{1}}^{* n} \cdot \mathbf{1}$. 
From the construction of $\mu^{n}\left(\pi, z_{\tilde{m}}, c, \mu\right)$ it follows

$$
\begin{gathered}
\frac{r_{\mathbf{1}}}{1+r_{\mathbf{1}}} \cdot \widetilde{M}_{\mathbf{1}}^{* n}+\pi_{\mathbf{1}}^{* n} \cdot\left(\frac{1}{1+r_{\mathbf{1}}} \square \tau_{\mathbf{1}}^{n}\right)+c^{* n} \sum_{\sigma \in S} \mu_{\sigma}^{* n}\left(\max _{s^{\prime} \in S} \bar{H}_{s^{\prime}}-\bar{H}_{\sigma}\right) \\
=\frac{\frac{r_{s}}{1+r_{s}} \widetilde{M}_{s}^{* n}+\frac{1}{1+r_{s}} \pi_{s}^{* n} \cdot \tau_{s}^{n}+c^{* n} \mu_{s}^{* n}\left(\max _{s^{\prime} \in S} \bar{H}_{s^{\prime}}-\bar{H}_{s}\right)}{\mu_{s}^{* n}} .
\end{gathered}
$$

Use this equation and (28) to get

$\frac{r_{s}}{1+r_{s}} \widetilde{M}_{s}^{* n}+\frac{1}{1+r_{s}} \pi_{s}^{* n} \cdot \tau_{s}^{n}-\widetilde{H}_{s}^{* n}+\mu_{s}^{* n} r_{0} \widetilde{M}_{0}^{* n}+\mu_{s}^{* n} \pi_{0}^{* n} \cdot \tau_{0}^{n}=\mu_{s}^{* n} \widetilde{H}_{0}^{* n}\left(1+r_{0}\right)$,

which proves that (26) holds.

\subsubsection{The limit of the fixed points for $n \rightarrow+\infty$}

Since $\left(\pi^{* n}, c^{* n}, \mu^{* n}, \widetilde{H}^{* n}, z^{* n}\right)$ is bounded for each $n$, we can let $n \rightarrow+\infty$ and choose a subsequence having a limit $\left(\pi^{*}, c^{*}, \mu^{*}, \widetilde{H}^{*}, z^{*}\right)$. We obviously have $\left(\pi^{*}, \mu^{*}, z_{\widetilde{m}}^{*}\right) \ll+\infty$, but $z_{x}^{*}$ could be infinite. Clearly, $\tau^{n} \rightarrow \tau$. By continuity, $\widetilde{H}^{*}=H\left(c^{*}, \mu^{*}\right), c^{*}=c\left(\pi^{*}, z_{\widetilde{m}}^{*}, \mu^{*}\right)$ and $\mu^{*}=\mu\left(\pi^{*}, c^{*}, \mu^{*}, z_{\widetilde{m}}^{*}\right)$. From $z_{\widetilde{m}}^{*} \ll+\infty$ we know that $c^{*}<+\infty$. From this we infer $\widetilde{H}^{*} \ll+\infty$. It remains to argue that markets clear, (26) holds, $0 \ll\left(\pi^{*}, c^{*}, \mu^{*}\right), z_{x}^{*} \ll+\infty$ and $z^{*} \in Z\left(\pi^{*}, \mu^{*}, \widetilde{H}^{*}\right)$.

Given the construction of $c^{n}(\pi, \widetilde{M}, \widetilde{H})$, Lemma (3.3) applies for every $n$. Together with money market clearing (the central bank accommodates money demand $)$ it hence follows $\left(1+r_{0}\right) \pi_{0}^{* n} \cdot\left(z_{x_{0}}^{* n}-\sum_{i} e_{0}^{i}\right)+\pi_{\mathbf{1}}^{* n} \cdot\left(z_{x_{\mathbf{1}}}^{* n}-\sum_{i} e_{\mathbf{1}}^{i}\right)$ $=0$ for every $n$. Hence

$$
\left(1+r_{0}\right) \pi_{0}^{*} \cdot\left(z_{x_{0}}^{*}-\sum_{i} e_{0}^{i}\right)+\pi_{\mathbf{1}}^{*} \cdot\left(z_{x_{\mathbf{1}}}^{*}-\sum_{i} e_{\mathbf{1}}^{i}\right)=0 .
$$

Since the interest rates are always finite, Lemma (1.8) allows for the application of the Lemmas (2.4) and (2.5). Hence, $z_{x}^{*}=\sum_{i} e^{i} \ll+\infty$ and $\pi^{*} \gg 0$.

From what we argued above, it follows easily that (26) is also true in the limit. $z^{*} \in Z\left(\pi^{*}, \mu^{*}, \widetilde{H}^{*}\right)$ follows from finite interest rates and $\pi^{*} \gg 0$ since Lemma (1.3) and Lemma (2.2) apply. Therefore, we need only to show that $c^{*}>0$ and $\mu^{*} \gg 0$. 
We first prove that $\mu^{*} \gg 0$. Suppose $\mu_{s}^{*}=0$ for $s \in S$. From the definition of $\mu(\pi, \widetilde{m}, c, \mu)$ we get $z_{\widetilde{m}_{s}}=0$ and $\tau_{s}=0$. For every $n$ along the sequence of fixed points, the consumer's budget in $s \in S$ is

$$
\begin{aligned}
\pi_{s}^{* n} \cdot x_{s}^{i * n}+\frac{\widetilde{b}_{s}^{i * n}}{1+r_{s}}+\widetilde{m}_{s}^{i * n} & \leq \pi_{s}^{* n} \cdot e_{s}^{i}+\left(\widetilde{b}_{0}^{i * n}+A_{s} \widetilde{\theta}^{i * n}+\widetilde{m}_{0}^{i * n}\right) \mu_{s}^{* n}+\delta^{i} \widetilde{H}_{s}^{*} \\
\widetilde{m}_{s}^{i * n} & \geq \pi_{s}^{* n} \cdot\left(x_{s}^{i * n}-e_{s}^{i}\right)^{-} \\
\widetilde{b}_{s}^{i * n}+\widetilde{m}_{s}^{i * n} & =0 .
\end{aligned}
$$

Since households optimize, we must have $\frac{r_{s}}{1+r_{s}} \widetilde{m}_{s}^{i * n}=\frac{r_{s}}{1+r_{s}} \pi_{s}^{* n} \cdot\left(x_{s}^{i * n}-e_{s}^{i}\right)^{-}$. We can use this to derive the equivalent formulation

$$
\pi_{s}^{* n} \cdot\left(x_{s}^{i * n}-e_{s}^{i}\right)^{+}=\frac{\pi_{s}^{* n} \cdot\left(x_{s}^{i * n}-e_{s}^{i}\right)^{-}}{1+r_{s}}+\left(\widetilde{b}_{0}^{i * n}+A_{s} \widetilde{\theta}^{i * n}+\widetilde{m}_{0}^{i * n}\right) \mu_{s}^{* n}+\delta^{i} \widetilde{H}_{s}^{* n} .
$$

By the cash-in-advance constraint, $\left(x_{s}^{i *}-e_{s}^{i}\right)^{-}=0$ for every $i \in I$. Since markets clear and nobody sells goods it follows that $x_{s}^{i *}=e_{s}^{i}$ for all $i \in I$. From $\widetilde{H}(c, \mu)$, we know that $\widetilde{H}_{s}^{*}=0$. Hence we get $\left(\widetilde{b}_{0}^{i *}+A_{s} \widetilde{\theta}^{i *}+\widetilde{m}_{0}^{i *}\right) \mu_{s}^{*}=0$ from the budget constraint. For $n \rightarrow \infty$, we get from the continuity of the budget set and from what we said previously that

$$
\pi_{s}^{*} \cdot\left(x_{s}^{i *}-e_{s}^{i}\right)^{+}=\frac{\pi_{s}^{*} \cdot\left(x_{s}^{i *}-e_{s}^{i}\right)^{-}}{1+r_{s}} .
$$

Define, for every $i \in I$, the utility function $v^{i}: \mathbb{R}_{+}^{L} \rightarrow \mathbb{R}$ by $v^{i}\left(\zeta_{s}^{i}\right):=$ $u^{i}\left(x_{0}^{i *}, x_{1}^{i *}, \ldots, e_{s}^{i}+\zeta_{s}^{i}, \ldots, x_{S}^{i *}\right)$. From what we said before, it follows $0=$ $\arg \max \left\{v^{i}\left(\zeta_{s}^{i}\right) \mid \pi_{s}^{*} \cdot \zeta_{s}^{i+} \leq \frac{\pi_{s}^{*} \cdot \zeta_{s}^{i-}}{1+r_{s}}\right\}, \forall i \in I$. Define the function $\bar{\zeta}_{s}\left(\zeta_{s}^{i}, r_{s}\right)$ : $\mathbb{R}^{L} \times \mathbb{R}_{+} \rightarrow \mathbb{R}^{L}$ by

$$
\bar{\zeta}_{s l}\left(\zeta_{s}^{i}, r_{s}\right):=\left\{\begin{array}{ll}
\zeta_{s l}^{i} & \text { if } \zeta_{s l}^{i}<0 \\
\frac{\zeta_{s l}^{i}}{1+r_{s}} & \text { otherwise }
\end{array}, \forall l \in L,\right.
$$

and a utility function $\bar{v}_{r_{s}}^{i}\left(\zeta_{s}^{i}\right):=v^{i}\left(\bar{\zeta}_{s}\left(\zeta_{s}^{i}, r_{s}\right)\right)$. As argued in Dubey and Geanakoplos (1992, pp. 418-419) we then get the equivalence that $0=$ $\arg \max \left\{v^{i}\left(\zeta_{s}^{i}\right) \mid \pi_{s}^{*} \cdot \zeta_{s}^{i+} \leq \frac{\pi_{s}^{*} \cdot \zeta_{s}^{i-}}{1+r_{s}}\right\}$ if and only if $0=\arg \max \left\{\bar{v}_{r_{s}}^{i}\left(\zeta_{s}^{i}\right) \mid \pi_{s}^{*}\right.$. $\left.\zeta_{s}^{i+} \leq \pi_{s}^{*} \cdot \zeta_{s}^{i-}\right\}$. If we consider an economy with $I$ agents having concave utilities $\bar{v}_{r_{s}}^{i}\left(\zeta_{s}^{i}\right)$ and endowments $e_{s}^{i}$, then no-trade is a Walrasian equilibrium for this economy at prices $\pi^{*}$. By Lemma 2 in Dubey and Geanakoplos (2003(a)), at the initial endowment allocation (in state $s \in S$ ) there are no gains to $r_{s}$-diminished trade. Hence, $r_{s} \geq \gamma_{s}\left(x_{-s}^{*}, e_{s}\right)$ from the definition of 
$\gamma_{s}\left(x_{-s}^{*}, e_{s}\right)$ - a contradiction to the Gains-to-Trade Hypothesis in Assumption 8. Therefore, we must have $\mu_{s}^{*}>0$ for every $s \in S$.

The definition of $c\left(\pi, z_{\widetilde{m}}, \widetilde{H}\right)$ and $\mu\left(\pi, z_{\widetilde{m}}, c, \widetilde{H}\right)$ now immediately imply $c^{*}>0$.

It follows as before that the limit of the fixed point vectors correspond to a monetary equilibrium in the abstract economy with interest rate peg and fixed transfers.

\subsection{Proof of Theorem 4}

This proof is a combination of the proofs of Theorems 2 and 3.

\subsubsection{Preliminary Definitions}

The augmented taxation $\tau^{n} \in \mathbb{R}_{+}^{(S+1) L}$, the government transfer function $\widetilde{H}(c, \mu):=\left(\widetilde{H}_{0}, \widetilde{H}_{1}, \ldots, \widetilde{H}_{S}\right)(c, \mu)$, the inverse price level function $c^{n}\left(\pi, r, \mu, z_{\widetilde{m}}\right)$ and the martingale-measure function $\mu^{n}\left(\pi, r, c, \mu, z_{\widetilde{m}}\right):=\left(\mu_{1}^{n}, \ldots, \mu_{S}^{n}\right)\left(\pi, r, c, \mu, z_{\widetilde{m}}\right)$ are defined as in Theorem 3. $c^{n}\left(\pi, r, \mu, z_{\widetilde{m}}\right)$ is a bounded and continuous function of $\left(\pi, r, \mu, z_{\widetilde{m}}\right)$ for each $n$ as long as $\bar{H}_{0}>0$ and $z_{\widetilde{m}} \ll+\infty$. Under the latter condition, $\mu^{n}\left(\pi, r, c, \mu, z_{\widetilde{m}}\right)$ is also bounded and continuous. By the construction of $\tau^{n}$, as long as $\pi \gg 0$ we have $c^{n}>0, \mu_{s}^{n}>0$ and $\sum_{s=1}^{S} \mu_{s}^{n}=1$ for all finite $n$.

\subsubsection{Construction of a fixed point mapping}

Define $\Delta^{n}$ and $\Omega^{n}$ as in Theorem 2 and denote aggregate demand with $Z^{n}(\pi, \mu, r, \widetilde{H}):=Z\left(\pi, \mu, r, \widetilde{H}, \tau^{n}\right)$. Lemma (3.4) allows us to define the compact and convex set $K_{\widetilde{m}}$ such that $K_{\widetilde{m}} \supseteq Z_{\widetilde{m}}^{n}(\pi, \mu, r, \widetilde{H})$ for all $\pi \in \Delta$, $\mu \in \Delta^{S-1}, r \in \Omega^{n}$ and $\widetilde{H} \in \mathbb{R}_{+}^{S+1}$. As argued above, for positive $\bar{H}_{0}$ we can define a compact and convex set $K_{c}^{n}$ such that $K_{c}^{n} \supseteq c^{n}\left(\pi, r, \mu, z_{\widetilde{m}}\right)$ for all $\pi \in \Delta^{n}, r \in \Omega^{n}, \mu \in \Delta^{S-1}$ and $z_{\widetilde{m}} \in K_{\widetilde{m}} . \mu^{n}\left(\pi, r, c, \mu, z_{\widetilde{m}}\right)$ lies in a compact and convex set $K_{\mu}^{n} \subset \operatorname{interior}\left(\Delta^{S-1}\right)$ for $\pi \in \Delta^{n}, z_{\widetilde{m}} \in K_{\widetilde{m}}$, $\mu \in \Delta^{S-1}, c \in K_{c}^{n}$, and $z_{\widetilde{m}} \in K_{\widetilde{m}}$. Introduce the compact and convex set $K_{\widetilde{H}}^{n}$ such that $K_{\widetilde{H}}^{n} \supseteq \widetilde{H}(c, \mu)$ for all $c \in K_{c}^{n}$ and $\mu \in \Delta$. Further define the compact and convex set $K_{x}^{n}$ such that $K_{x}^{n} \supseteq Z_{x}^{n}(\pi, \mu, r, \widetilde{H})$ for all $\pi \in \Delta^{n}, \mu \in K_{\mu}^{n}, r \in \Omega^{n}$, and $\widetilde{H} \in K_{\widetilde{H}}^{n}$. Again, denote the product set by $K^{n}:=K_{x}^{n} \times K_{\widetilde{m}}$. Define the mapping

$f^{n}: \Delta^{n} \times \Omega^{n} \times K_{c}^{n} \times K_{\mu}^{n} \times K_{\widetilde{H}}^{n} \times K^{n} \rightrightarrows \Delta^{n} \times \Omega^{n} \times K_{c}^{n} \times K_{\mu}^{n} \times K_{\widetilde{H}}^{n} \times K^{n}$ 
by

$$
(\pi, r, c, \mu, \widetilde{H}, z) \stackrel{f^{n}}{\mapsto}\left(f_{\pi}^{n}, f_{r}^{n}, f_{c}^{n}, f_{\mu}^{n}, f_{\widetilde{H}}^{n}, f_{z}^{n}\right)
$$

where

$$
\begin{aligned}
f_{\pi}^{n}(\pi, r, c, \mu, \widetilde{H}, z) & :=\underset{\left\{\pi \in \Delta^{n}\right\}}{\arg \max }\left\{\left(1+r_{0}\right) \pi_{0} \cdot\left(z_{x_{0}}-\sum_{i} e_{0}^{i}\right)+\pi_{\mathbf{1}} \cdot\left(z_{x_{\mathbf{1}}}-\sum_{i} e_{\mathbf{1}}^{i}\right)\right\}, \\
f_{r}^{n}(\pi, r, c, \mu, \widetilde{H}, z) & :=\underset{r \in \Omega^{n}}{\arg \max }\left\{r_{0}\left(\frac{z_{\widetilde{m}_{0}}}{c}-\bar{M}_{0}\right)+\frac{r_{\mathbf{1}}}{1+r_{\mathbf{1}}} \cdot\left(z_{\widetilde{m}_{\mathbf{1}}} \square \frac{1}{c \mu}-\bar{M}_{\mathbf{1}}\right)\right\}, \\
f_{c}^{n}(\pi, r, c, \mu, \widetilde{H}, z) & :=c^{n}\left(\pi, r, \mu, z_{\widetilde{m}}\right), \\
f_{\mu}^{n}(\pi, r, c, \mu, \widetilde{H}, z) & :=\mu^{n}\left(\pi, r, c, \mu, z_{\widetilde{m}}\right), \\
f_{\widetilde{H}}^{n}(\pi, r, c, \mu, \widetilde{H}, z) & :=\widetilde{H}(c, \mu), \\
f_{z}^{n}(\pi, r, c, \mu, \widetilde{H}, z) & :=Z^{n}(\pi, r, \mu, \widetilde{H}),
\end{aligned}
$$

where $\frac{1}{c \mu}:=\left(\frac{1}{c \mu_{s}}\right)_{s \in S}$ in the second line. As before, there exists a fixed point $\left(\pi^{* n}, r^{* n}, c^{* n}, \mu^{* n}, \widetilde{H}^{* n}, z^{* n}\right)$ for every $n$.

\subsubsection{The limit of the fixed points is an equilibrium}

For $n \rightarrow \infty$, we get $\left(\pi^{* n}, r^{* n}, c^{* n}, \mu^{* n}, \widetilde{H}^{* n}, z^{* n}\right) \rightarrow\left(\pi^{*}, r^{*}, c^{*}, \mu^{*}, \widetilde{H}^{*}, z^{*}\right)$ and $\tau^{n} \rightarrow \tau$. We want to show that $\left(\pi^{*}, r^{*}, c^{*}, \mu^{*}, \widetilde{H}^{*}, z^{*}\right)$ is an equilibrium for the abstract economy with taxation $\tau$.

By the definitions of $c^{n}\left(\pi, r, \mu, z_{\widetilde{m}}\right)$ and $\mu^{n}\left(\pi, r, c, \mu, z_{\widetilde{m}}\right)$, we get for each $n$

$$
\begin{aligned}
\frac{1}{c^{* n} \mu_{s}^{* n}} \frac{r_{s}^{* n}}{1+r_{s}^{* n}} z_{\tilde{m}_{s}}^{* n}+\frac{1}{c^{* n} \mu_{s}^{* n}} \frac{1}{1+r_{s}^{* n}} \pi_{s}^{* n} \cdot \tau_{s}^{n} & +\frac{1}{c^{* n}}\left(r_{0}^{* n} z_{\tilde{m}_{0}}^{* n}+\pi_{0}^{* n} \cdot \tau_{0}^{n}\right) \\
& =\bar{H}_{0}\left(1+r_{0}^{* n}\right)+\bar{H}_{s},
\end{aligned}
$$

or alternatively

$$
\begin{array}{r}
\frac{1}{c^{* n} \mu_{s}^{* n}} \frac{r_{s}^{* n}}{1+r_{s}^{* n}} z_{\tilde{m}_{s}}^{* n}+\frac{1}{c^{* n} \mu_{s}^{* n}} \frac{1}{1+r_{s}^{* n}} \pi_{s}^{* n} \cdot \tau_{s}^{n}+\frac{1}{c^{* n}}\left(r_{0}^{* n} z_{\bar{m}_{0}}^{* n}+\pi_{0}^{* n} \cdot \tau_{0}^{n}\right) \\
-r_{0}^{* n} \bar{H}_{0}=\bar{H}_{0}+\bar{H}_{s}
\end{array}
$$


From this,

$$
\begin{aligned}
\frac{r_{s}^{* n}}{1+r_{s}^{* n}}\left(\frac{1}{c^{* n} \mu_{s}^{* n}} z_{\bar{m}_{s}}^{* n}-\right. & \left.\bar{M}_{s}\right)+r_{0}^{* n}\left(\frac{1}{c^{* n}} z_{\bar{m}_{0}}^{* n}-\bar{M}_{0}\right) \\
& +r_{0}^{* n}\left(\bar{M}_{0}-\bar{H}_{0}\right)<\bar{H}_{0}+\bar{H}_{s}-\frac{r_{s}^{* n}}{1+r_{s}^{* n}} \bar{M}_{s},
\end{aligned}
$$

from which we infer

$$
\begin{aligned}
& \lim _{n \rightarrow \infty} \frac{r_{s}^{* n}}{1+r_{s}^{* n}}\left(\frac{1}{c^{* n} \mu_{s}^{* n}} z_{\tilde{m}_{s}}^{* n}-\bar{M}_{s}\right)+\lim _{n \rightarrow \infty} r_{0}^{* n}\left(\frac{1}{c^{* n}} z_{\tilde{m}_{0}}^{* n}-\bar{M}_{0}\right) \\
& \quad+\lim _{n \rightarrow \infty} r_{0}^{* n}\left(\bar{M}_{0}-\bar{H}_{0}\right) \leq \bar{H}_{0}+\bar{H}_{s}-\frac{r_{s}^{*}}{1+r_{s}^{*}} \bar{M}_{s} .
\end{aligned}
$$

By the construction of $f_{r}^{n}$ and the fact that $\bar{M}_{0}>\bar{H}_{0}$, we get in the limit

$$
\begin{aligned}
\lim _{n \rightarrow \infty} r_{0}^{* n}\left(\frac{1}{c^{* n}} z_{\tilde{m}_{0}}^{* n}-\bar{M}_{0}\right) & \geq 0 \\
\lim _{n \rightarrow \infty} \frac{1}{c^{* n} \mu_{s}^{* n}} \frac{r_{s}^{* n}}{1+r_{s}^{* n}} z_{\tilde{m}_{s}}^{* n} & \geq \frac{r_{s}^{*}}{1+r_{s}^{*}} \bar{M}_{s}, \\
\lim _{n \rightarrow \infty} r_{0}^{* n}\left(\bar{M}_{0}-\bar{H}_{0}\right) & \geq 0 .
\end{aligned}
$$

Therefore, we have

$$
r_{0}^{*}<+\infty,
$$

since otherwise $\lim _{n \rightarrow \infty} r_{0}^{* n}\left(\bar{M}_{0}-\bar{H}_{0}\right)=+\infty$, contradicting the inequality. In addition, since $\frac{r_{s}^{*}}{1+r_{s}^{*}} \bar{M}_{s} \leq \bar{H}_{0}+\bar{H}_{s}$, we know that

$$
r_{s}^{*}<\frac{\bar{H}_{0}+\bar{H}_{s}}{\bar{M}_{s}-\bar{H}_{0}-\bar{H}_{s}}<+\infty .
$$

From $r \ll+\infty$ and the construction of $f_{r}^{n}$ we can infer that

$$
\begin{aligned}
\lim _{n \rightarrow \infty} \frac{1}{c^{* n}} z_{\widetilde{m}_{0}}^{* n} & \leq \bar{M}_{0}, \\
\lim _{n \rightarrow \infty} \frac{1}{c^{* n} \mu_{s}^{* n}} z_{\tilde{m}_{s}}^{* n} & \leq \bar{M}_{s},
\end{aligned}
$$

which further implies

$$
\begin{aligned}
\lim _{n \rightarrow \infty} r_{0}^{* n}\left(\frac{1}{c^{* n}} z_{\tilde{m}_{0}}^{* n}-\bar{M}_{0}\right) & =0, \\
\lim _{n \rightarrow \infty} \frac{r_{s}^{* n}}{1+r_{s}^{* n}}\left(\frac{1}{c^{* n} \mu_{s}^{* n}} z_{\tilde{m}_{s}}^{* n}-\bar{M}_{s}\right) & =0 .
\end{aligned}
$$


From the definition of $c^{n}\left(\pi, r, \mu, z_{\widetilde{m}}\right)$ and $\widetilde{H}(c, \mu)$ we get

$r_{0} z_{\tilde{m}_{0}}^{* n}+\frac{r_{\mathbf{1}}}{1+r_{\mathbf{1}}} \cdot z_{\widetilde{m}_{\mathbf{1}}}^{* n}+\pi_{0}^{* n} \cdot \tau_{0}^{n}+\pi_{\mathbf{1}}^{* n} \cdot\left(\frac{1}{1+r_{\mathbf{1}}} \square \tau_{\mathbf{1}}^{n}\right)=\widetilde{H}_{0}^{* n}\left(1+r_{0}\right)+\widetilde{H}_{\mathbf{1}}^{* n} \cdot \mathbf{1}$.

Adding up the intertemporal individual budget sets over all households and plugging in this equation gives for every $n$

$$
\left(1+r_{0}^{* n}\right) \pi_{0}^{* n} \cdot\left(z_{x_{0}}^{* n}-\sum_{i} e_{0}^{i}\right)+\pi_{\mathbf{1}}^{* n} \cdot\left(z_{x_{1}}^{* n}-\sum_{i} e_{\mathbf{1}}^{i}\right)=0 .
$$

The left hand side of this equation is just the commodity price player's objective function. In the limit we get

$$
\left(1+r_{0}^{*}\right) \pi_{0}^{*} \cdot\left(z_{x_{0}}^{*}-\sum_{i} e_{0}^{i}\right)+\pi_{\mathbf{1}}^{*} \cdot\left(z_{x_{\mathbf{1}}}^{*}-\sum_{i} e_{\mathbf{1}}^{i}\right)=0 .
$$

Given this, it is easy to see that the commodity markets clear. From Lemma (2.4) we get $\pi^{*} \gg 0$. Hence we have $z^{*} \in Z\left(\pi^{*}, r^{*}, \mu^{*}, \widetilde{H}^{*}\right)$.

From the construction of $c^{n}\left(\pi, r, \mu, z_{\widetilde{m}}\right)$, we know that $c^{*}<+\infty$. Next, we show that $c^{*}>0$ and $\mu^{*} \gg 0$.

For $\mu^{*} \gg 0$, the argument is quite similar to the one given in the Theorem 3. For every $s \in S$, if $\tau_{s}>0$, it is obvious that $\mu_{s}^{*}>0$. Suppose $\tau_{s}=0$ for some $s \in S$ and $\mu_{s}^{*}=0$. From the fact $c^{*}<+\infty$, we know that $\lim _{n \rightarrow \infty} c^{* n} \mu_{s}^{* n}=0$. From the inequality $\lim _{n \rightarrow \infty} \frac{1}{c^{* n} \mu_{s}^{* n}} z_{\tilde{m}_{s}}^{* n} \leq \bar{M}_{s}$, we know that $z_{\tilde{m}_{s}}^{*}=0$ (otherwise $\lim _{n \rightarrow \infty} \frac{1}{c^{* n} \mu_{s}^{* n}} z_{\tilde{m}_{s}}^{* n}=+\infty>\bar{M}_{s}$ ). Therefore, the argument in the proof of Theorem 3 applies, which means $\gamma_{s}\left(x_{-s}^{*}, e_{s}\right) \leq r_{s}^{*}$. Hence, by $(29), \gamma_{s}\left(x_{-s}^{*}, e_{s}\right)<\frac{\bar{H}_{0}+\bar{H}_{s}}{\bar{M}_{s}-\bar{H}_{0}-\bar{H}_{s}}$, a contradiction to the Gains-toTrade hypothesis in Assumption 9. Therefore, we must have $\mu_{s}^{*}>0$ for all $s \in S$.

The result of $c^{*}>0$ can be proved in a similar way as in Theorem 3 .

Given $\mu^{*} \gg 0$ and $c^{*}>0$ we can now infer from equations (30) - (33) that the money markets clear.

It follows that the limit of the fixed point vectors corresponds to a monetary equilibrium in the abstract economy with money supply control and fixed transfers. 


\section{References}

[1] Balasko, Y., Cass, D., 1989. The structure of financial equilibrium with exogenous yields: The case of incomplete markets. Econometrica 57, 135-162.

[2] Bloise, G., 2006. A note on the existence of monetary equilibrium over an infinite horizon, Economic Theory 27, 59-77.

[3] Bloise, G., Dréze, J. H., Polemarchakis, H.M., 2005. Monetary equilibria over an infinite horizon. Economic Theory 25, 51-74.

[4] Bloise, G., Polemarchakis, H.M., 2006. Theory and practice of monetary policy. Economic Theory 27, 1-23.

[5] Cass, D., 1984. Competitive equilibrium with incomplete financial markets. CARESS working paper 84-09 (University of Pennsylvania).

[6] Dréze, J.H., Polemarchakis, H.M., 2000. Monetary equilibria. in: G. Debreu, W. Neuefeind and W. Trockel, eds., Economic Essays: A Festschrift in Honor of W. Hildenbrand (Springer Verlag) p.p.83-108.

[7] Dubey, P., Geanakoplos, J.D., 1992. The value of money in a finite horizon Economy: A role for banks, in: P. Dasgupta, D. Gale, D. Hart, and E. Maskin, eds., Economic Analysis of Markets and Games: Essays in Honor of Frank Hahn. Cambridge, MA: MIT Press, pp. 407-444.

[8] Dubey, P., Geanakoplos, J.D., 2003(a). Inside and outside fiat money, gains to trade, and IS-LM. Economic Theory 21, 347-397.

[9] Dubey, P., Geanakoplos, J.D., 2003(b). Monetary equilibrium with missing markets. Journal of Mathematical Economics 39, 585-618.

[10] Dubey, P., Geanakoplos, J.D., 2006. Determinacy with nominal assets and outside money. Economic Theory 27, 79-106.

[11] Duffie, D., Shafer, W., 1985. Equilibrium in incomplete market I: Basic model of generic existence. Journal of Mathematical Economics 14, 285300 .

[12] Geanakoplos, J., Mas-Colell, A., 1989. Real indeterminacy with finanacial assets. Journal of Economic Theory 47(1), 22-38. 
[13] Gourdel, P., Triki, L., 2005. Incomplete markets and monetary policy. Working paper B05024, CERMSEM, Université Paris PanthéonSorbonne.

[14] Harrison, M., Kreps, D., 1979, Martingales and arbitrage in multiperiod security markets, Journal of Economic Theory 20, 381 - 408.

[15] Nakajima, T., Polemarchakis, H.M., 2001. Money and prices under uncertainty. Working paper No. 01-32, Brown University.

[16] Nakajima, T., Polemarchakis, H.M., 2005. Money and prices under uncertainty. Review of Economic Studies 72, 223-246.

[17] Svensson, L., 1985. Money and asset prices in a cash-in-advance economy. Journal of Political Economy 93, 919-944.

[18] Werner, J., 1985. Equilibrium in economies with incomplete financial markets. Journal of Economic Theory 36, 110-119.

[19] Woodford, M., 1994. Monetary policy and price level determinacy in a cash-in-advance economy. Economic Theory 4, 345-380.

[20] Woodford, M., 1995. Price-level determinacy without control of a monetary aggregate, Carnegie-Rochester Conference Series on Public Policy $43,1-46$.

[21] Woodford, M., 2001. Fiscal requirements for price stability, Journal of Money, Credit and Banking 33(3), 669-728. 